\title{
Highly Enantioselective Organocatalytic Cyclopropanation of Enals using Benzyl Chlorides
}

\author{
Marta Meazza, ${ }^{\dagger, \oplus}$ Maria Ashe, ${ }^{\dagger,}$ Hun Yi Shin,,${ }^{\dagger+}$ Hye Sung Yang, ${ }^{\dagger,+}$ Andrea Mazzanti, ${ }^{\S}$ Jung \\ Woon Yang, ${ }^{*}+, *$ and Ramon Rios ${ }^{*}, \dagger$, \\ $\dagger$ Faculty of Natural and Environmental Science, School of Chemistry, University of Southampton, Highfield \\ Campus, Southampton SO17 1BJ, United Kingdom \\ ¥Department of Energy Science, Sungkyunkwan University, Suwon 440-746, South Korea \\ $\S$ Department of Industrial Chemistry “Toso Montanari', School of Science, Alma Mater Studiorum - \\ University of Bologna, V. Risorgimento 4, 40136 Bologna, Italy
}

\begin{abstract}
Herein we describe the first enantioselective cyclopropanation of enals using benzyl chlorides as bifunctional (nucleophilic and electrophilic) reagents. The reaction is simply catalyzed by chiral secondary amines to afford the formyl cyclopropane derivatives in good yields and with moderate to excellent stereoselectivities.

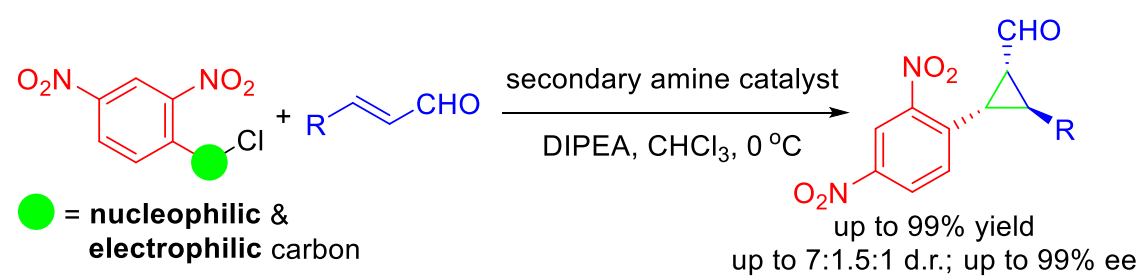

\section{- INTRODUCTION}

Cyclopropane subunits have always fascinated organic chemists because of their unusual structural properties and their wide presence in natural products and pharmaceuticals. The cyclopropane skeletal structure is often found in terpenes, pheromones, fatty acid metabolites, and unnatural amino acids; moreover, its derivatives present a plethora of biological activities such as insecticidal, antibiotic, antifungal, antitumor, and antiviral properties. For these reasons, many scientists are interested in developing new enantioselective methods for the construction of cyclopropanes.

Since the seminal report of Simmons and Smith on the reaction of alkenes with diiodomethane in the presence of zinc dust to afford cyclopropanes in high yields, ${ }^{1}$ several asymmetric versions of cyclopropanations were reported. These methodologies rely either on the use of stoichiometric amounts of chiral auxiliaries or promoters with allylic alcohols (or amines), $\alpha, \beta$ - 
unsaturated carbonyls, allenic alcohols, homoallylic ethers, or unfunctionalized alkenes, or on catalytic amounts of chiral transition-metal complexes with electron-deficient diazo compounds. Among these, the most common approach employs transition-metals (e.g., copper, rhodium, ruthenium and cobalt) to catalyze the reaction of diazoacetates with alkenes, rendering the final cyclopropanes in excellent results. ${ }^{2}$

During the past few years, organocatalysis has emerged as the third pillar of asymmetric organic catalysis, complementing the previous organometallic and enzymatic catalysis. Since the pioneering works of List and MacMillan in $2000,{ }^{3}$ many great accomplishments, including the design of new organocatalysts, strategies, and methodologies, have been achieved.

Recognizing the value of cyclopropanes, several research groups (e.g., Gaunt, ${ }^{4}$ Connon, ${ }^{5}$ MacMillan, ${ }^{6}$ Córdova, ${ }^{7}$ and $\mathrm{Wang}^{8}$ ) reported various organocatalytic methodologies. Most of them are based on the Michael-initiated ring-closing (MIRC) reaction of pre-enolized or readily enolizable nucleophilic species, such as $\alpha$-brominated malonates, bromonitromethanes and sulfur ylides, with unsaturated derivatives (e.g., enals, enones, and nitrostyrenes). In 2011, Lattanzi's group reported the asymmetric cyclopropanation via a domino Michael-alkylation reaction of alkenes bearing electron-withdrawing groups (EWGs), such as 2-arylidene-1,3indandiones, for the synthesis of spirocyclopropanes. ${ }^{9}$ Despite the impressive advances in this field, pre-enolized or enolizable compounds are still considered the most effective reactants. Very recently, aryl methanes and their derivatives, usually considered as poor nucleophiles, have been independently reported by the groups of Wang, ${ }^{10}$ Jørgensen ${ }^{11}$ and Lee ${ }^{12}$ as suitable nucleophilic reagents in the organocatalytic Michael addition to $\alpha, \beta$-unsaturated aldehydes (Scheme 1, Eq. 1). Their nucleophilicity is dramatically enhanced by the introduction of nitro groups at the ortho- and/or para-positions of the aromatic ring, as a results of strong inductive and resonance effects.

Moreover, our group investigated the enantioselective addition of alkylbenzoxazoles, acting as pseudo-benzylic functionalities, to enals, via a synergistic catalysis between organocatalysis and transition-metal catalysis (Scheme 1, Eq. 2); ${ }^{13}$ the coordination of palladium with the nitrogen atom of the benzoxazole moiety enhanced the nucleophilicity of the pseudo-benzylic position of $\alpha$-azaarenes. Recently, Melchiorre and co-workers reported the use of 2,4dinitrobenzyl bromide as a radical source for alkylation reactions (Scheme 1, Eq. 3). ${ }^{14}$ 
Scheme 1. Aryl Methane Derivatives Serving as Pseudo-Benzylic Functionalities

Previous works:

a) Wang et al., Jørgensen et al., and Lee et al.

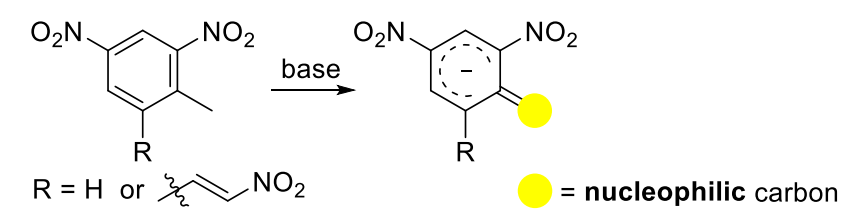

b) Rios et al.

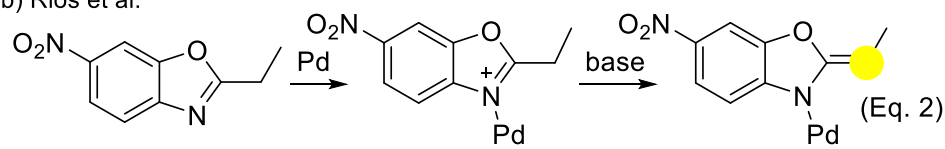

c) Melchiorre et al.

$\mathrm{O}_{2}^{\mathrm{O}_{2}}$

$=$ nucleophilic $\&$ electrophilic carbon

(Eq. 4)

On the basis of these previous reports and our experience with pseudo-benzylic functionalized molecules, we envisioned an easy access to cyclopropanes via an asymmetric organocatalytic Michael addition- $\alpha$-alkylation cascade of benzyl halides with enals. ${ }^{[15]}$ In general, benzyl halides are considered electrophilic species; however, when decorated with strong electronwithdrawing group (EWG), such as $-\mathrm{NO}_{2}$ group, on the aromatic rings and in the presence of a weak base, they could act as nucleophiles [Scheme 1, Eq. (4)]. The in-situ generation of a carbanion at the $\alpha$-position of the benzyl halide could initiate the organocascade reaction, ${ }^{[16]}$ in which the $\alpha, \beta$-unsaturated aldehyde, activated by an aminocatalyst, would act as electrophilic counterpart; the following irreversible intramolecular alkylation would give the benzylic cyclopropanated products by iminium and enamine activation.

\section{- RESULTS AND DISCUSSION}

In order to assess the feasibility of the proposed transformation, we commenced our studies by testing the reaction of 2,4-dinitrobenzyl chloride 1a and crotonaldehyde $\mathbf{2 a}$ and by evaluating the effect of parameters such as catalysts, solvents, bases, and temperature. As shown in Table 1 , the desired cyclopropane was initially obtained in $29 \%$ conversion with reasonable diastereo- 
and excellent enantioselectivity under illustrated reaction conditions $[20 \mathrm{~mol} \%$ of diphenylprolinol silyl ether catalyst I, 1.1 equiv. of 2,6-lutidine, toluene at room temperature] (Table 1, entry 1). The use of other solvents such as acetonitrile and chloroform results in a slight increase in the cyclopropane yield (Table 1, entries 2 and 4), and no reaction occurred when DMSO was used as the solvent (Table 1, entries 3). Next, we focused on the screening of bases, such as $\mathrm{K}_{2} \mathrm{CO}_{3}, \mathrm{Et}_{3} \mathrm{~N}$ and DIPEA ( $N, N$-diisopropylethylamine; Hunig's base); the presence of a base is crucial for trapping $\mathrm{HCl}$ released, thereby enhancing the reaction rate and reducing the formation of side products. Among various bases examined, DIPEA gave the full conversion when the reaction was performed with $20 \mathrm{~mol} \%$ of catalyst $\mathrm{I}$ in $\mathrm{CHCl}_{3}$ at room temperature (Table 1, entry 7). Next, several organocatalysts (II-V) were tested: di(trifluoromethyl)-substituted prolinol silyl ether II gave excellent enantioselectivity but low conversion, whereas proline III gave full conversion but low enantioselectivity (Table 1, entries 8-9).

Table 1. Optimization of the Asymmetric Cyclopropanation ${ }^{a}$

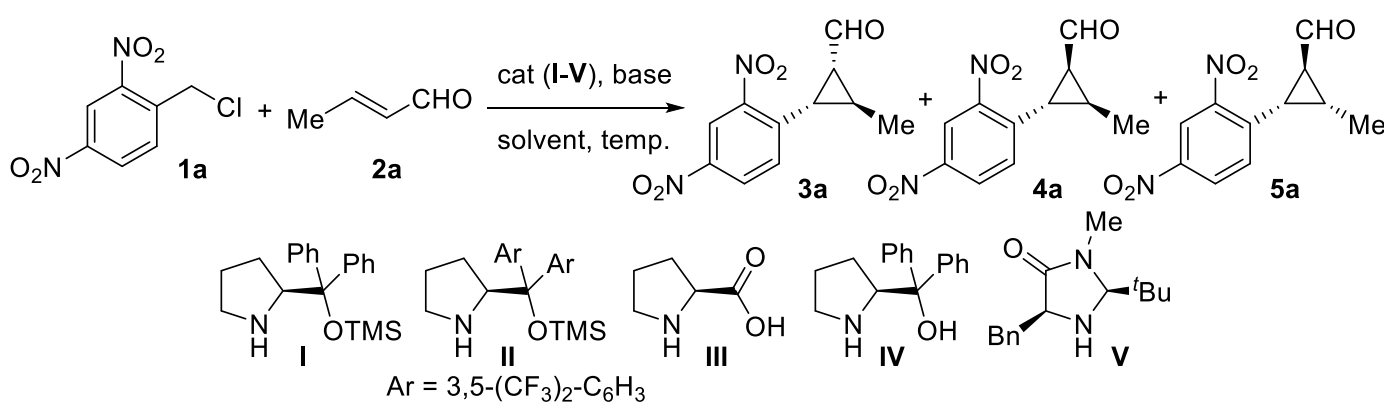

\begin{tabular}{|c|c|c|c|c|c|c|c|}
\hline entry & catalyst & solvent & base & temp. $\left({ }^{\circ} \mathrm{C}\right)$ & conv. $(\%)^{b}$ & $\begin{array}{l}\text { dr of } \\
\mathbf{3 a}: \mathbf{4} \mathbf{a}: 5 \mathbf{a}^{b}\end{array}$ & $\begin{array}{l}\text { ee of } \\
\mathbf{3 a}: \mathbf{4 a}: \mathbf{5 a}\end{array}$ \\
\hline 1 & I & Toluene & 2,6-Lutidine & $\mathrm{rt}$ & 29 & $7: 8: 1$ & $94: 87: 40$ \\
\hline 2 & I & $\mathrm{CH}_{3} \mathrm{CN}$ & 2,6-Lutidine & $\mathrm{rt}$ & 36 & $2: 2: 1$ & $93: 74: 76$ \\
\hline 3 & I & DMSO & 2,6-Lutidine & $\mathrm{rt}$ & - & - & - \\
\hline 4 & I & $\mathrm{CHCl}_{3}$ & 2,6-Lutidine & $\mathrm{rt}$ & 47 & $4: 2: 1$ & $98: 77: 67$ \\
\hline 5 & I & $\mathrm{CHCl}_{3}$ & $\mathrm{Et}_{3} \mathrm{~N}$ & $\mathrm{rt}$ & 71 & $3: 2: 1$ & $98: 92: 69$ \\
\hline 6 & I & $\mathrm{CHCl}_{3}$ & $\mathrm{~K}_{2} \mathrm{CO}_{3}$ & $\mathrm{rt}$ & 72 & 2.6:2:1 & 98:91:71 \\
\hline 7 & I & $\mathrm{CHCl}_{3}$ & DIPEA & $\mathrm{rt}$ & $>99$ & $4: 3: 1$ & $98: 77: 71$ \\
\hline 8 & II & $\mathrm{CHCl}_{3}$ & DIPEA & $\mathrm{rt}$ & 27 & $2.4: 2.2: 1$ & $98: 71: 31$ \\
\hline 9 & III & $\mathrm{CHCl}_{3}$ & DIPEA & $\mathrm{rt}$ & $>99$ & $2: 2: 1$ & $59: 44: 21$ \\
\hline 10 & IV & $\mathrm{CHCl}_{3}$ & DIPEA & $\mathrm{rt}$ & trace & - & - \\
\hline 11 & $\mathrm{~V}$ & $\mathrm{CHCl}_{3}$ & DIPEA & $\mathrm{rt}$ & trace & - & - \\
\hline 12 & I & $\mathrm{CHCl}_{3}$ & DIPEA & 0 & $>99$ & $3: 2.5: 1$ & $98: 81: 66$ \\
\hline
\end{tabular}


$13 \mathrm{I} \quad \mathrm{CHCl}_{3} \quad$ DIPEA $\quad-20 \quad>99 \quad 3: 2.5: 1 \quad 98: 82: 40$

${ }^{a}$ General reaction conditions: 1a (1 equiv), 2a (2 equiv), catalysts I-V (20 mol\%), base $(1.1 \mathrm{mmol})$, solvent, temperature ( 0 or rt). ${ }^{b}$ Determined by ${ }^{1} \mathrm{H}$ NMR of the crude reaction. ${ }^{c}$ Determined by HPLC analysis using a chiral column.

Surprisingly, diphenyl prolinol IV and MacMillan's second-generation imidazolidinone catalyst $\mathbf{V}$, which has been previously used by Lattanzi and co-workers in similar MIRC reactions with excellent results, did not catalyze the reaction to any significant extent, yielding only a trace amount of product (Table 1, entries 10-11). We also investigated the effect of the reaction temperature on the yield and selectivity. When the reaction was performed at $0{ }^{\circ} \mathrm{C}$, the stereoselectivity slightly increased without any loss of catalytic activity (Table 1, entry 12). A similar result was achieved when the reaction was carried out at $-20{ }^{\circ} \mathrm{C}$ (Table 1 , entry 13). Thus, the best conditions for the asymmetric cyclopropanation reaction are as follows: 20 mol\% of chiral organocatalyst $\mathbf{I}, 1.1$ equiv. of DIPEA, $\mathrm{CHCl}_{3}$ at $0{ }^{\circ} \mathrm{C}$.

With the optimal conditions in hands, we extended the reaction scope to various benzyl chlorides 1 bearing strong electron-withdrawing groups (EWGs) with both aliphatic and aromatic $\alpha, \beta$-unsaturated aldehydes 2 (Table 2). In the reaction of aliphatic $\alpha, \beta$-unsaturated aldehydes with benzyl chloride 1a, the corresponding products were produced in high yields with excellent diastereo- and enantioselectivities of major products 3 (Table 2, entries 1-5).

Table 2. Substrate Scope for the Asymmetric Cyclopropanation ${ }^{a}$

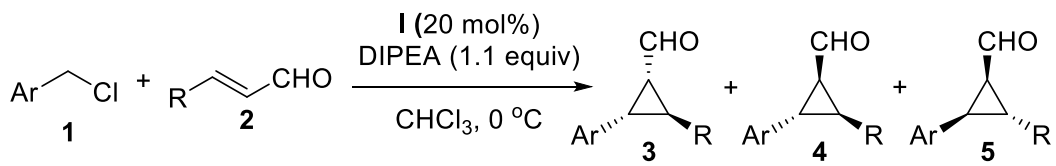

\begin{tabular}{|c|c|c|c|c|c|c|}
\hline entry & $\mathrm{Ar}$ & $\mathrm{R}$ & product (major) & yield $(\%)^{b}$ & dr of $3: 4: 5^{c}$ & ee of $\mathbf{3 : 4 : 5 ^ { d }}$ \\
\hline 1 & & $\mathrm{Me}$ & & 61 & $8: 3: 1$ & 98:73:60 \\
\hline
\end{tabular}


$2^{e}$<smiles>CCCc1ccc([N+](=O)[O-])cc1[N+](=O)[O-]</smiles>

3<smiles>[X]c1ccc([N+](=O)[O-])cc1[N+](=O)[O-]</smiles>

4<smiles>[X]c1c([N+](=O)[O-])ccc2cc([N+](=O)[O-])ccc12</smiles>

5

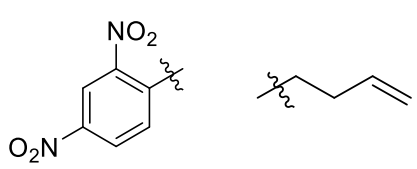

6<smiles>[X]c1ccc([N+](=O)[O-])cc1[N+](=O)[O-]</smiles>

$\mathrm{CO}_{2} \mathrm{Et}$

7<smiles>O=[N+]([O-])c1ccc2cccc([N+](=O)[O-])c2c1</smiles>

$\mathrm{Ph}$

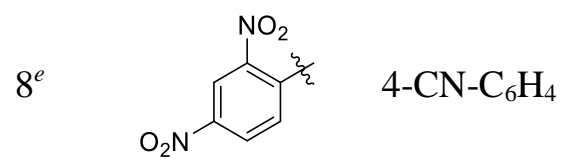

9<smiles>[X]c1ccc([N+](=O)[O-])cc1[N+](=O)[O-]</smiles>

4- $\mathrm{NO}_{2}-\mathrm{C}_{6} \mathrm{H}_{4}$

10<smiles>[Y]c1c([14CH3])ccc2c1ccc([N+](=O)[O-])[n+]2[O-]</smiles>

11<smiles>[X]c1ccc([N+](=O)[O-])cc1[N+](=O)[O-]</smiles>

4-Cl- $\mathrm{C}_{6} \mathrm{H}_{4}$<smiles>[X]c1ccc([N+](=O)[O-])cc1[N+](=O)[O-]</smiles>

4-Br- $\mathrm{C}_{6} \mathrm{H}_{4}$
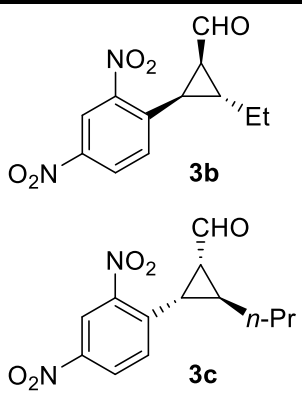

$\mathrm{CHO}$<smiles>CCC1C(C=O)C1c1ccc([N+](=O)[O-])cc1[N+](=O)[O-]</smiles><smiles>C=CCCC1C(C=O)[C@H]1c1ccc([N+](=O)[O-])cc1[N+](=O)[O-]</smiles><smiles>CCOC(=O)C1C(C=O)C1c1ccc([N+](=O)[O-])cc1[N+](=O)[O-]</smiles><smiles>O=CC1C(c2ccccc2)[C@H]1c1ccc([N+](=O)[O-])cc1[N+](=O)[O-]</smiles><smiles>N#Cc1ccc(C2C(C=O)C2c2ccc([N+](=O)[O-])cc2[N+](=O)[O-])cc1</smiles><smiles>O=CC1C(c2ccc([N+](=O)[O-])cc2)[C@@H]1c1ccc([N+](=O)[O-])cc1[N+](=O)[O-]</smiles><smiles>C[C@]1(c2ccc([N+](=O)[O-])cc2[N+](=O)[O-])C(C=O)C1c1ccc(F)cc1</smiles><smiles>O=C[C@H]1C(c2ccc(Cl)cc2)[C@H]1c1ccc([N+](=O)[O-])cc1[N+](=O)[O-]</smiles><smiles>O=CC1C(c2ccc(Br)cc2)C1c1ccc([N+](=O)[O-])cc1[N+](=O)[O-]</smiles>

99

66

68

78

80

67

$9: 2: 1$

99:93:n.d.

82

$7: 1: 1$

99:93:54

$7: 1.5: 1 \quad 99: 92: 54$

4:1:1 99:92:n.d.

$1: 1: 1 \quad 73: 40: 80$

$3: 2: 1 \quad 97: 91: 96$

2:1.5:1 99:99:n.d

2:2:1 98:n.d.:n.d.

78

1.5:1.5:1 98:91:90

86

2.5:1.5:1 99:94:97

71

$2: 2: 1$

99:95:98 
$13^{e}$<smiles>[X]c1ccc([N+](=O)[O-])cc1[N+](=O)[O-]</smiles>

4-Me- $\mathrm{C}_{6} \mathrm{H}_{4}$

14<smiles>Cc1ccc([N+](=O)[O-])cc1[N+](=O)[O-]</smiles>

4- $\mathrm{MeO}-\mathrm{C}_{6} \mathrm{H}_{4}$<smiles>[X]c1ccc(C(F)(F)F)cc1[N+](=O)[O-]</smiles>

$\mathrm{Ph}$

$15^{f}$

$16^{e, f}$<smiles>Cc1ccc(C(F)(F)F)cc1[N+](=O)[O-]</smiles><smiles>Cc1ccc([C@@H]2[C@H](C=O)[C@H]2c2ccc([N+](=O)[O-])cc2[N+](=O)[O-])cc1</smiles>

98:90:94

a Reaction conditions: 1 (1 equiv), 2 (2 equiv), catalysts I (20 mol\%), DIPEA (1.1 equiv), C $\mathrm{HCl}_{3}$, temperature $\left(0{ }^{\circ} \mathrm{C}\right), 3-5 \mathrm{~h}$. ${ }^{b}$ Isolated yield (sum of diastereomers). ${ }^{c}$ Determined by ${ }^{1} \mathrm{H} \mathrm{N}$ MR of the crudereaction. ${ }^{d}$ Determined by HPLC analysis using a chiral column. ${ }^{e}$ Using ent-I $\left[(R)\right.$-configuration) ${ }^{f}$ Run at $60{ }^{\circ} \mathrm{C}$. [n.d. = not determined].

The reaction with glyoxylate derivatives gave a 1:1:1 mixture of diastereomers with moderate enantioselectivities (Table 2, entry 6). Next, we studied the reaction with aromatic enals, and investigated the influence of the electronic properties of the aromatic substituents on the reactivity and selectivity. In almost all cases, the final products were obtained in excellent enantioselectivities with moderate diastereoselectivities (Table 2, entries 7-16). Aromatic $\alpha, \beta$ unsaturated aldehydes bearing electron-donating groups (EDGs), such as -OMe or -Me group, gave yields and stereoselectivities similar to those obtained for enals with relatively stronger EWGs, such as $-\mathrm{CN}$ and $-\mathrm{NO}_{2}$ group (Table 2, entries 8-9 and 13-14). In the presence of halogen substituents on the aromatic ring of the $\alpha, \beta$-unsaturated aldehyde, similar yields and stereoselectivities are observed for all compounds studied, and the desired products are obtained in very good yields, with moderate diastereoselectivities and excellent enantioselectivities (Table 2, entries 10-12). Finally, we tested the reaction of substrates bearing other aromatic substituents and it was found that the cyclopropanation reaction requires two strong EWGs on the aromatic ring of the benzylic chloride in order to enhance the acidity and nucleophilicity of the benzylic position. 4-Trifluoromethyl-2-nitrobenzyl chloride reacted with enals to give the corresponding chiral cyclopropanes in moderate yields and 
diastereoselectivities and good enantioselectivities (Table 2, entries 15-16). However, this reaction requires higher reaction temperatures $\left(60^{\circ} \mathrm{C}\right)$, which could be responsible for the lower stereoselectivities obtained as compared with those observed for dinitrobenzyl derivatives. A plausible catalytic cycle for the asymmetric cyclopropanation is depicted in Scheme 2.

Scheme 2. Plausible Catalytic Cycle for the Enantioselective Intermolecular Cyclopropanation

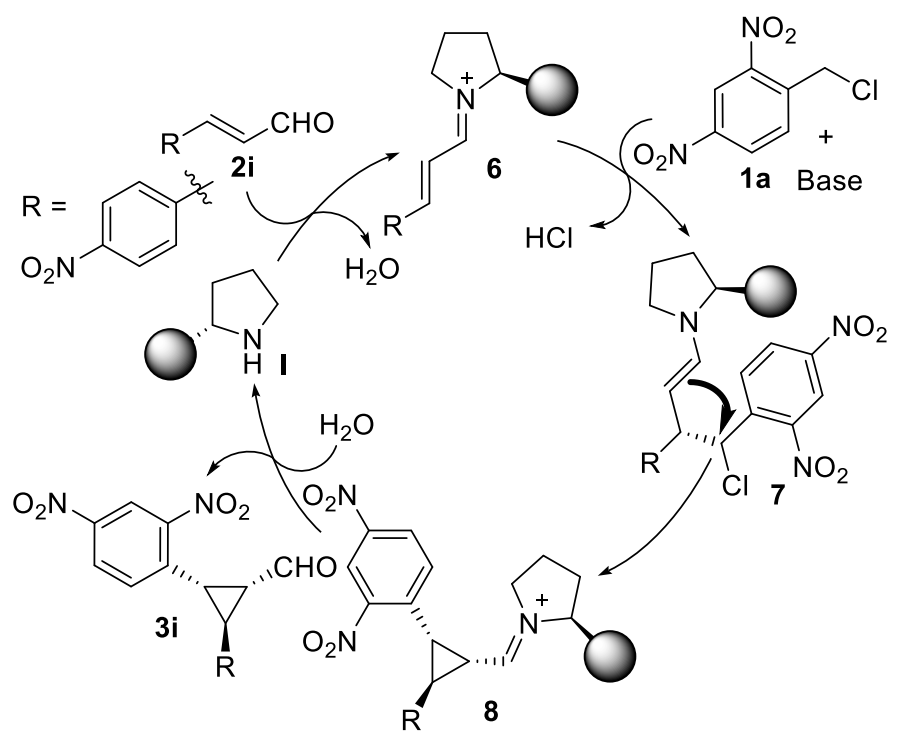

$\alpha, \beta$-unsaturated iminium ion $\mathbf{6}$ was initially formed by the reaction of diphenyl prolinol silyl ether catalyst $\mathbf{I}$ with $\alpha, \beta$-unsaturated aldehyde $2 \mathbf{i}$. At this stage, the bulky group of catalyst $\mathbf{I}$ shields the $\mathrm{Si}$-face of $\alpha, \beta$-unsaturated iminium ion $\mathbf{6}$. Intermediate $\mathbf{8}$ was therefore formed by nucleophilic attack of 1a predominantly on the $R e$-face of iminium ion 6 via Michael addition, followed by intramolecular ring-closing reaction between the enamine and the secondary alkyl chloride. Iminium ion $\mathbf{8}$ is hydrolyzed to the desired product $\mathbf{3 i}$ and catalyst $\mathbf{I}$ is regenerated.

Absolute stereochemistry of product ent-3i, which is derived from $(R)$-configured catalyst (ent-I), was unambiguously determined by single crystal X-ray diffraction analysis (Figure 1).

Figure 1. X-ray Structure of Cyclopropanation Product ent-3i 


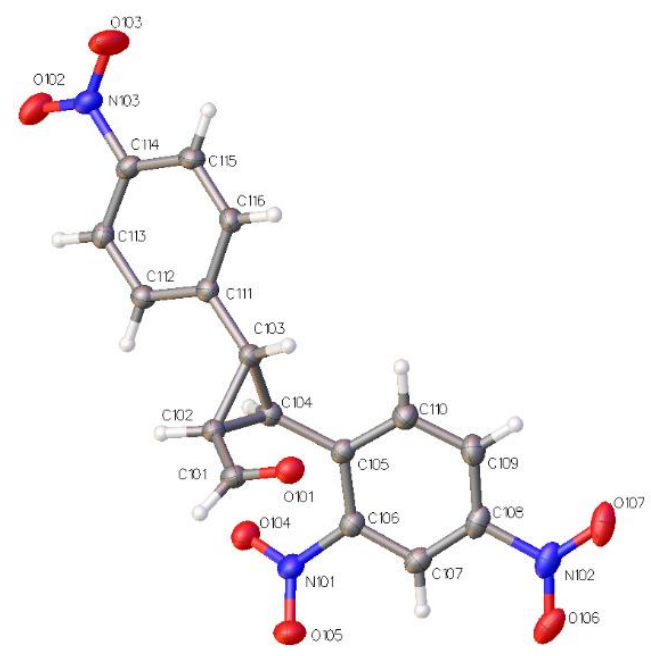

Ellipsoids are shown at the $50 \%$ probability level.

The relative configuration of compounds $\mathbf{3}, \mathbf{4}$, and $\mathbf{5}$ was determined by NMR analysis and the absolute configuration was established by circular dichroism (CD) spectroscopy (see Supporting Information). In order to confirm the absolute configuration of compound 4, we envisioned the ring-opening of cyclopropanes $\mathbf{3 g}$ and $\mathbf{4 g}$ via $N$-heterocyclic carbene (NHC) catalysis.

Selective ring-opening of cyclopropane derivatives is an important issue in organic synthesis because it is always associated with inherent regiochemical preferences, which are highly dependent on the nature of the functional groups on the cyclopropanes. In general, exceptionally regioselective outcomes are observed when strong EWGs (i.e., ketones, esters, and amides) are located in a vicinal position to EDGs. In 2006, Bode and co-workers reported the redox-esterification of formylcyclopropanes with alcohol, thiol or water, using NHC catalysis (Scheme 3). ${ }^{17}$ In all cases, stronger EWGs such as ketones, esters, amides, or nitro groups are essential to obtain regioselective ring-opened products with high yields.

Scheme 3. NHC-Catalyzed Ring-Opening of Cyclopropanes Bearing Strong Electron-Withdrawing Groups 


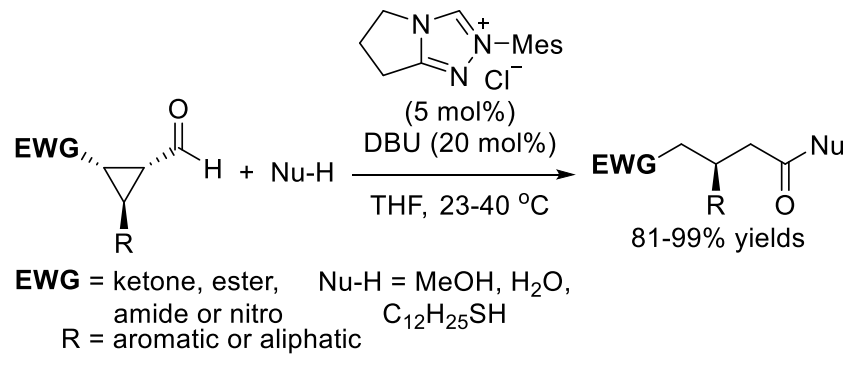

Based on these results, we became interested in the ring-opening reaction of cyclopropane bearing two aryls (3g), instead of two electronically different functional groups, on the cyclopropane ring through NHC catalysis. As a result, only a single product $\mathbf{1 0}$ was obtained under our experimental conditions [cyclopropanated product $\mathbf{3 g}$, $\mathrm{MeOH}$ (3 equiv.), thiazolium precatalyst 9 (20 mol\%), DIPEA (40 mol\%), $\mathrm{CH}_{2} \mathrm{Cl}_{2}(0.33 \mathrm{M})$, rt.] (Scheme 4).

Scheme 4. Regioselective Ring-Opening of Cyclopropanes $\mathbf{3 g}$ and $\mathbf{4 g}$.

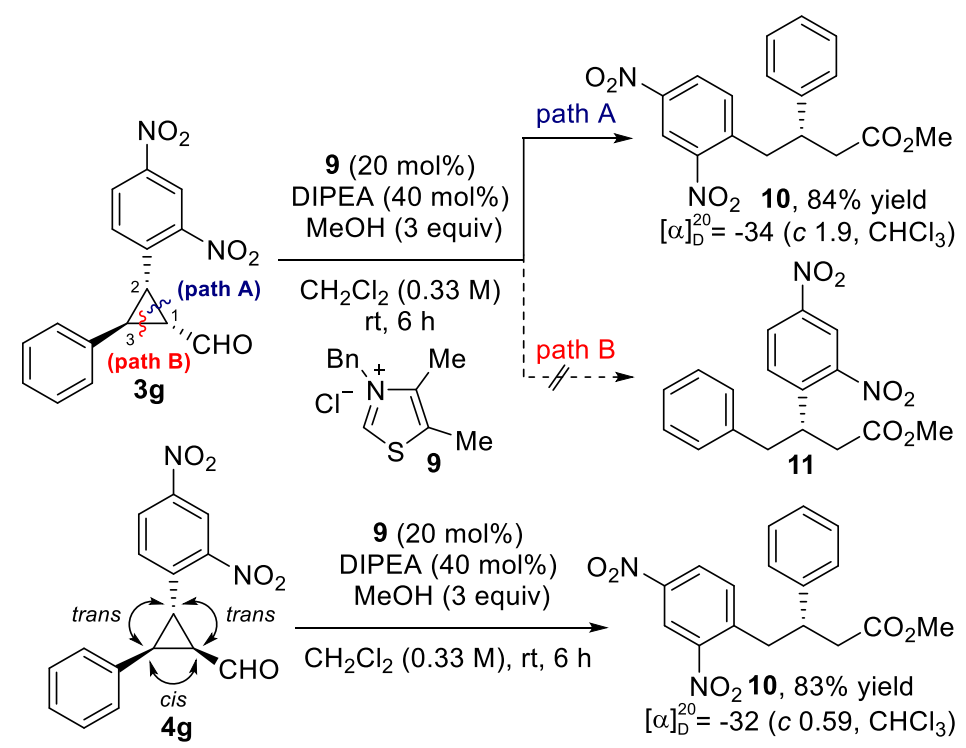

Moreover, we performed the reaction with the second major diastereomer $\mathbf{4 g}$ under the same conditions, and the same configuration of product $\mathbf{1 0}$ was observed, as confirmed by comparison of the optical rotation with that of the product derived from $\mathbf{3 g}$. Thus, the absolute configuration of compound $\mathbf{4 g}$ was ultimately determined on the basis of optical rotation 
measurements and NMR analysis.

Finally, we propose a plausible mechanism, to elucidate the origin of the regioselectivity of this process (Scheme 5). At first, the nucleophilic addition of the NHC to the aldehyde produced enaminol 13, the so-called Breslow intermediate. At this stage, two competitive pathways for the ring-opening reaction are possible, and control could be achieved by finetuning the electronic properties of the substituents on two aromatic rings. When the $\mathrm{C} 2$ position is relatively more electron-deficient, this $\mathrm{C}-\mathrm{C}$ bond is preferentially cleaved because two nitro groups on the aromatic ring can stabilize the resultant carbanion 14. Thus, compound $\mathbf{1 0}$ was regioselectively formed instead of compound $\mathbf{1 1 .}$

Scheme 5. Rationale for the Origin of the Regioselectivity

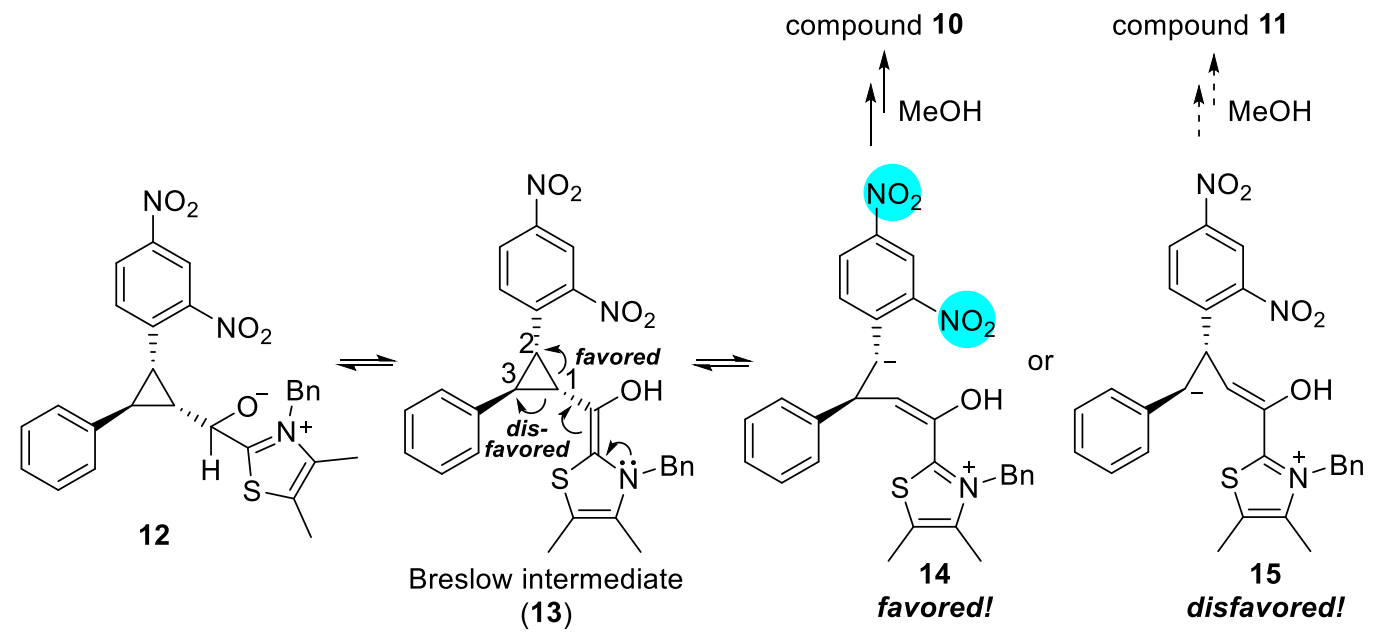

To the best of our knowledge, this is the first example of regioselective ring-opening reaction of cyclopropanes containing two aromatic rings. This approach is a formal benzyl addition to unsaturated esters, which can be achieved in good yields and excellent enantioselectivities. This two-step procedure is particularly advantageous for the synthesis of aliphatic derivatives that can only be obtained in low yields using Wang and Jørgensen's methodologies. ${ }^{10,11}$

\section{- CONCLUSION}

In summary, we reported the first cyclopropanation reaction of benzyl chlorides with enals. 
The final products were obtained in good yields and enantioselectivities and with moderate to excellent diastereoselectivities. Moreover, we also demonstrated the ring-opening of diarylsubstituted cyclopropanes furnished the desired products with complete regioselective fashion through N-heterocyclic carbene catalysis.

\section{- EXPERIMENTAL SECTION}

Thin layer chromatography (TLC) was performed on Merck TLC Silicagel $60 \mathrm{~F}_{254}$. Product spots were visualized by UV-light at $254 \mathrm{~nm}$. Column chromatography was effectuated using silica gel (Geduran Si60, 40-63 $\mu \mathrm{m}$ ). Infra-red spectra were recorded on a Nicolet 280 FT-IR; the IR analyses were performed as a liquid IR with the compounds dissolved in $\mathrm{CHCl}_{3} .{ }^{1} \mathrm{H}$ NMR, ${ }^{13} \mathrm{C}$ NMR, ${ }^{19} \mathrm{~F}$ NMR were recorded with a Bruker DPX400 NMR. High resolution mass spectra were recorded using a MaXis (Bruker Daltonics, Bremen, Germany) mass spectrometer equipped with a Time of Flight (TOF) analyzer.

General procedure for asymmetric cyclopropanation using 2,4-dinitrobenzyl chloride (A): In a vial, $(S)$ - $\alpha, \alpha$-diphenylprolinol trimethylsilyl ether $(20 \mathrm{~mol} \%)$ and 2,4-dinitrobenzyl chloride (1 equiv., $100 \mathrm{mg}, 0.4617 \mathrm{mmol})$ were dissolved in chloroform $(1 \mathrm{~mL})$. The vial was covered with aluminum foil and the resulting solution was cooled to $0{ }^{\circ} \mathrm{C}$. After 15 minutes, $\alpha, \beta$-unsaturated aldehyde (2 equiv.) and $N, N$-diisopropylethylamine (DIPEA, 1.1 equiv.) were added to the reaction mixtures. The resulting solution was stirred at $0{ }^{\circ} \mathrm{C}$ for $3-5$ hours. After the reaction was completed, the crude product was purified by flash column chromatography (hexane/EtOAc) to obtain the desired cyclopropane.

General procedure for asymmetric cyclopropanation using 2-nitro-4-(trifluoromethyl)benzyl chloride (B): In a vial, (S)- $\alpha, \alpha$-diphenylprolinol trimethylsilyl ether (20 mol\%) and 2-nitro-4(trifluoromethyl)-benzyl chloride (1 equiv., $100 \mathrm{mg}, 0.417 \mathrm{mmol}$ ) were dissolved in chloroform ( $1 \mathrm{~mL})$. The vial was covered with aluminum foil and $\alpha, \beta$-unsaturated aldehyde ( 2 equiv.) and $\mathrm{N}, \mathrm{N}$-diisopropylethylamine (DIPEA, 1.1 equiv.) were added to the reaction mixtures. The resulting solution was stirred at $60{ }^{\circ} \mathrm{C}$ for 24 hours. After the reaction was completed, the crude was purified by flash column chromatography (hexane/EtOAc) to obtain the desired cyclopropane. 
2-(2,4-Dinitrophenyl)-3-methylcyclopropane-1-carbaldehyde (3a, 4a and 5a): The title compound was synthesized according to general procedure A. The product was purified by column chromatography (hexane/EtOAc $=10: 1$ ), affording the title compound as a yellow oil; Diastereomeric ratios of 3a:4a:5a $=8: 3: 1$; Enantiomeric exceses of 3a:4a:5a $=98: 73: 60$; Total yield of 3a:4a:5a $=61 \%(71 \mathrm{mg})$.

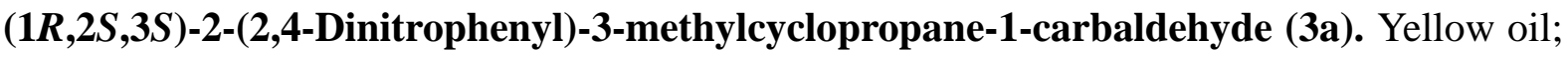
The enantiomeric excess was determined by HPLC using a Chiralpak OD-H column (hexane/ $i$ $\operatorname{PrOH}=80: 20$, flow rate $=1.0 \mathrm{~mL} / \mathrm{min}, \lambda=230 \mathrm{~nm}): \mathrm{t}_{\mathrm{R}}=21.4 \mathrm{~min}, \mathrm{t}_{\mathrm{R}}=24.6 \mathrm{~min} ;[\alpha]_{\mathrm{D}}{ }^{26}=-$ $90.8^{\circ}\left(c\right.$ 0.6, $\left.\mathrm{CHCl}_{3}\right)(\boldsymbol{S}$ catalyst $) ;{ }^{1} \mathrm{H} \mathrm{NMR}\left(400 \mathrm{MHz}, \mathrm{CDCl}_{3}\right) \delta=9.43(\mathrm{~d}, J=2.8,1 \mathrm{H}), 8.71$ $(\mathrm{d}, J=2.3,1 \mathrm{H}), 8.36(\mathrm{dd}, J=8.6,2.3,1 \mathrm{H}), 7.66(\mathrm{~d}, J=8.6,1 \mathrm{H}), 2.86(\mathrm{dd}, J=8.1,8.0,1 \mathrm{H})$, $2.46(\mathrm{ddd}, J=8.0,4.8,2.8,1 \mathrm{H}), 2.18(\mathrm{~m}, 1 \mathrm{H}), 1.38(\mathrm{~d}, J=6.0,1 \mathrm{H}) ;{ }^{13} \mathrm{C}$ NMR $(101 \mathrm{MHz}$, $\left.\mathrm{CDCl}_{3}\right) \delta=198.1(\mathrm{CHO}), 150.1(\mathrm{Cq}), 146.8(\mathrm{Cq}), 138.8(\mathrm{Cq}), 133.6(\mathrm{CH}), 126.7(\mathrm{CH}), 119.9$ $(\mathrm{CH}), 38.0(\mathrm{CH}), 34.2(\mathrm{CH}), 24.2(\mathrm{CH}), 17.5\left(\mathrm{CH}_{3}\right)$; IR $v_{\max }\left(\mathrm{KBr}, \mathrm{cm}^{-1}\right)$ : 3105, 2966, 2866, 2009, 1702, 1604, 1530 (aromatic $\mathrm{NO}_{2}$ ), 1461, 1346 (aromatic $\mathrm{NO}_{2}$ ), 1152, 1087, 1044, 938, 909, 854, 835, 737; HRMS (ESI) calcd for $\mathrm{C}_{11} \mathrm{H}_{10} \mathrm{~N}_{2} \mathrm{O}_{5}[\mathrm{M}+\mathrm{Na}]^{+} 273.0482$, found 273.0480.

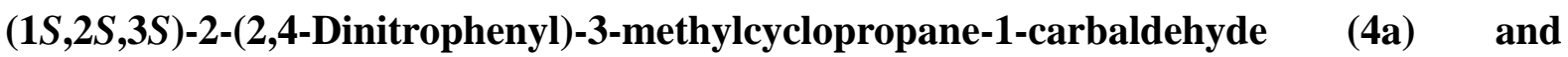
(1S,2S,3R)-2-(2,4-dinitrophenyl)-3-methylcyclopropane-1-carbaldehyde (5a).

[inseparable mixture of diastereoisomers]: Yellow oil; The enantiomeric excess of product $\mathbf{4 a}$ was determined by HPLC using a Chiralpak OD-H column (hexane $/ i$-PrOH $=80: 20$, flow rate $=1.0 \mathrm{~mL} / \mathrm{min}, \lambda=230 \mathrm{~nm}$ ): $\mathrm{t}_{\mathrm{R}}=29.7 \mathrm{~min}, \mathrm{t}_{\mathrm{R}}=27.3 \mathrm{~min}$; The enantiomeric excess of product 5a was determined by HPLC using a Chiralpak OD-H column (hexane/i-PrOH = 80:20, flow rate $=1.0 \mathrm{~mL} / \mathrm{min}, \lambda=230 \mathrm{~nm}): \mathrm{t}_{\mathrm{R}}=43.4 \mathrm{~min}, \mathrm{t}_{\mathrm{R}}=37.0 \mathrm{~min} ;{ }^{1} \mathrm{H} \mathrm{NMR}\left(400 \mathrm{MHz}, \mathrm{CDCl}_{3}\right)$ $\left[\right.$ diastereomer $-\mathrm{H}^{\prime}(\mathbf{4 a})$; diastereomer $\left.-\mathrm{H}(\mathbf{5 a})\right] \delta=9.69\left(\mathrm{~d}, J=3.9,1 \mathrm{H}^{\prime}\right), 9.45(\mathrm{~d}, J=4.2,1 \mathrm{H})$, $8.82(\mathrm{~d}, J=2.3,1 \mathrm{H}), 8.76\left(\mathrm{~d}, J=2.3,1 \mathrm{H}^{\prime}\right), 8.41(\mathrm{dd}, J=8.5,2.3,1 \mathrm{H}), 8.36(\mathrm{dd}, J=8.6,2.3$, $\left.1 \mathrm{H}^{\prime}\right), 7.59(\mathrm{~d}, J=8.5,1 \mathrm{H}), 7.40\left(\mathrm{~d}, J=8.6,1 \mathrm{H}^{\prime}\right), 3.28(\mathrm{dd}, J=9.8,5.5,1 \mathrm{H}), 3.18(\mathrm{dd}, J=6.2$, 5.9, 1H'), $2.36(\mathrm{ddd}, J=9.2,4.9,4.1,1 \mathrm{H}), 2.20-2.14(\mathrm{~m}, 1 \mathrm{H}), 2.11$ (ddd, $J=8.6,6.0,3.9$, $\left.1 \mathrm{H}^{\prime}\right), 1.96-1.84\left(\mathrm{~m}, 1 \mathrm{H}^{\prime}\right), 1.43\left(\mathrm{~d}, J=6.3,3 \mathrm{H}^{\prime}\right), 0.88(\mathrm{~d}, J=6.2,3 \mathrm{H}) ;{ }^{13} \mathrm{C} \mathrm{NMR}(101 \mathrm{MHz}$, $\mathrm{CDCl}_{3}$ ) [diastereomer (4a) - C'; diastereomer (5a) - C] $\delta=198.3(\mathrm{CHO}), 197.9\left(\mathrm{C}^{\prime} \mathrm{HO}\right), 150.1$ $(\mathrm{Cq}), 146.6\left(\mathrm{Cq}^{\prime}\right), 141.3\left(\mathrm{Cq}^{\prime}\right), 138.2\left(\mathrm{Cq}^{\prime}\right), 134.7(\mathrm{Cq}), 133.2(\mathrm{CH}), 131.2(\mathrm{Cq}), 130.0\left(\mathrm{C}^{\prime} \mathrm{H}\right)$, $127.2\left(\mathrm{C}^{\prime} \mathrm{H}\right), 127.0(\mathrm{CH}), 120.3(\mathrm{CH}), 120.3\left(\mathrm{C}^{\prime} \mathrm{H}\right), 37.2(\mathrm{CH}), 36.4\left(\mathrm{C}^{\prime} \mathrm{H}\right), 29.8\left(\mathrm{C}^{\prime} \mathrm{H}\right), 29.6$ $(\mathrm{CH}), 27.5\left(\mathrm{C}^{\prime} \mathrm{H}\right), 23.8(\mathrm{CH}), 12.9\left(\mathrm{CH}_{3}\right), 12.7\left(\mathrm{C}^{\prime} \mathrm{H}_{3}\right)$; IR $v_{\max }\left(\mathrm{KBr}, \mathrm{cm}^{-1}\right):$ 3105, 2966, 2866, 2009, 1702, 1604, 1530 (aromatic $\mathrm{NO}_{2}$ ), 1461, 1346 (aromatic $\mathrm{NO}_{2}$ ), 1152, 1087, 1044, 938, 
909, 854, 835, 737; HRMS (ESI) calcd for $\mathrm{C}_{11} \mathrm{H}_{10} \mathrm{~N}_{2} \mathrm{O}_{5}[\mathrm{M}+\mathrm{Na}]^{+} 273.0482$, found 273.0476 .

2-(2,4-Dinitrophenyl)-3-ethylcyclopropane-1-carbaldehyde (3b, $4 \mathrm{~b}$ and 5b): The title compound was synthesized according to general procedure A. The product was purified by column chromatography (hexane/EtOAc $=10: 1$ ), affording the title compound as a yellow oil; Diastereomeric ratios of $\mathbf{3 b} \mathbf{4} \mathbf{4 b}: \mathbf{5 b}=$ 9:2:1; Enantiomeric exceses of 3b:4b:5b = 99:93:n.d.; Total yield of $\mathbf{3 b}: \mathbf{4 b}: \mathbf{5 b}=68 \%(83.3 \mathrm{mg})$.

$(1 S, 2 R, 3 R)$-2-(2,4-Dinitrophenyl)-3-ethylcyclopropane-1-carbaldehyde (3b). Yellow oil; The enantiomeric excess was determined by HPLC using a Chiralpak OD-H column (hexane/ $i$ $\mathrm{PrOH}=80: 20$, flow rate $=1.0 \mathrm{~mL} / \mathrm{min}, \lambda=230 \mathrm{~nm}): \mathrm{t}_{\mathrm{R}}=18.1 \mathrm{~min}, \mathrm{t}_{\mathrm{R}}=19.6 \mathrm{~min} ;[\alpha]_{\mathrm{D}}{ }^{21}=-$ $61.9^{\circ}\left(c 1.2, \mathrm{CHCl}_{3}\right)(\boldsymbol{S}$ catalyst $),[\alpha]_{\mathrm{D}}{ }^{21}=+58.9^{\circ}\left(c\right.$ 1.3, $\left.\mathrm{CHCl}_{3}\right)$ ( $\boldsymbol{R}$ catalyst $){ }^{1} \mathrm{H} \mathrm{NMR}(400$ $\left.\mathrm{MHz}, \mathrm{CDCl}_{3}\right) \delta=9.45(\mathrm{~d}, J=2.7,1 \mathrm{H}), 8.72(\mathrm{~d}, J=2.3,1 \mathrm{H}), 8.37(\mathrm{dd}, J=8.6,2.3,1 \mathrm{H}), 7.68$ $(\mathrm{d}, J=8.6,1 \mathrm{H}), 2.88(\mathrm{dd}, J=8.2,8.0,1 \mathrm{H}), 2.49(\mathrm{ddd}, J=8.1,4.9,2.7,1 \mathrm{H}), 2.19-2.11(\mathrm{~m}$, 1H), $1.72-1.54(\mathrm{~m}, 1 \mathrm{H}), 1.09$ (t, $J=7.4,3 \mathrm{H}) ;{ }^{13} \mathrm{C} \mathrm{NMR}\left(101 \mathrm{MHz}, \mathrm{CDCl}_{3}\right) \delta 198.0(\mathrm{CHO})$, $150.1(\mathrm{Cq}), 146.8(\mathrm{Cq}), 139.0(\mathrm{Cq}), 133.6(\mathrm{CH}), 126.8(\mathrm{CH}), 119.9(\mathrm{CH}), 36.8(\mathrm{CH}), 33.1(\mathrm{CH})$, $31.6(\mathrm{CH}), 25.6\left(\mathrm{CH}_{2}\right), 13.0\left(\mathrm{CH}_{3}\right)$; IR $v_{\max }\left(\mathrm{KBr}, \mathrm{cm}^{-1}\right)$ : 3099, 2963, 2927, 2853, 2022, 1701, 1605, 1530 (aromatic $\mathrm{NO}_{2}$ ), 1463, 1346 (aromatic $\mathrm{NO}_{2}$ ), 1150, 1067, 991, 907, 835, 738; HRMS (ESI) calcd for $\mathrm{C}_{12} \mathrm{H}_{12} \mathrm{~N}_{2} \mathrm{O}_{5}[\mathrm{M}+\mathrm{Na}]^{+} 287.0638$, found 287.0634 .

$(1 R, 2 R, 3 R)$-2-(2,4-Dinitrophenyl)-3-ethylcyclopropane-1-carbaldehyde (4b). Yellow oil; The enantiomeric excess was determined by HPLC using a Chiralpak OD-H column (hexane/i$\operatorname{PrOH}=80: 20$, flow rate $=1.0 \mathrm{~mL} / \mathrm{min}, \lambda=230 \mathrm{~nm}): \mathrm{t}_{\mathrm{R}}=33.5 \mathrm{~min}, \mathrm{t}_{\mathrm{R}}=19.3 \mathrm{~min} ;[\alpha]_{\mathrm{D}}{ }^{21}=-$ $27.6^{\circ}\left(c 0.5, \mathrm{CHCl}_{3}\right)\left(\boldsymbol{R}\right.$ catalyst); ${ }^{1} \mathrm{H} \mathrm{NMR}\left(400 \mathrm{MHz}, \mathrm{CDCl}_{3}\right) \delta=9.66(\mathrm{~d}, J=4.0,1 \mathrm{H}), 8.75$ $(\mathrm{d}, J=2.3,1 \mathrm{H}), 8.36(\mathrm{dd}, J=8.6,2.3,1 \mathrm{H}), 7.39(\mathrm{~d}, J=8.6,1 \mathrm{H}), 3.24(\mathrm{dd}, J=5.9,5.8,1 \mathrm{H})$, $2.33(\mathrm{ddd}, J=9.1,4.9,4.2,1 \mathrm{H}), 1.87$ (ddt, $J=13.7,8.9,6.8,2 \mathrm{H}), 1.72-1.62(\mathrm{~m}, 1 \mathrm{H}), 1.02(\mathrm{t}$, $J=7.2,3 \mathrm{H}) ;{ }^{13} \mathrm{C} \mathrm{NMR}\left(101 \mathrm{MHz}, \mathrm{CDCl}_{3}\right) \delta=197.6(\mathrm{CHO}), 150.1(\mathrm{Cq}), 146.5(\mathrm{Cq}), 141.4$ $(\mathrm{Cq}), 129.9(\mathrm{CH}), 127.2(\mathrm{CH}), 120.3(\mathrm{CH}), 36.4(\mathrm{CH}), 35.3(\mathrm{CH}), 29.0(\mathrm{CH}), 20.9\left(\mathrm{CH}_{2}\right), 13.9$ $\left(\mathrm{CH}_{3}\right)$; IR $v_{\max }\left(\mathrm{KBr}, \mathrm{cm}^{-1}\right): 3099,2963,2927,2853,2022,1701,1605,1530$ (aromatic $\mathrm{NO}_{2}$ ), 1463, 1346 (aromatic $\mathrm{NO}_{2}$ ), 1150, 1067, 991, 907, 835, 738; HRMS (ESI) calcd for $\mathrm{C}_{12} \mathrm{H}_{12} \mathrm{~N}_{2} \mathrm{O}_{5}[\mathrm{M}+\mathrm{Na}]^{+}$287.0638, found 287.0640.

$(1 R, 2 R, 3 S)$-2-(2,4-Dinitrophenyl)-3-ethylcyclopropane-1-carbaldehyde (5b). The title compound was unable to characterize becasue of extremely low yield, which is attributed to high diastereoselectivity of the major diastereomers. 
2-(2,4-Dinitrophenyl)-3-propylcyclopropane-1-carbaldehyde (3c, 4c and 5c): The title compound was synthesized according to general procedure A. The product was purified by column chromatography (hexane/EtOAc $=7: 1$ ), affording the title compound as a yellow oil; Diastereomeric ratios of $\mathbf{3 c}: \mathbf{4 c}: \mathbf{5} \mathbf{c}=7: 1: 1$; Enantiomeric exceses of $\mathbf{3 c}: \mathbf{4} \mathbf{c}: \mathbf{5} \mathbf{c}=99: 93: 54$; Total yield of $\mathbf{3 c}: 4 \mathbf{c}: 5 \mathbf{c}=82 \%(105 \mathrm{mg})$.

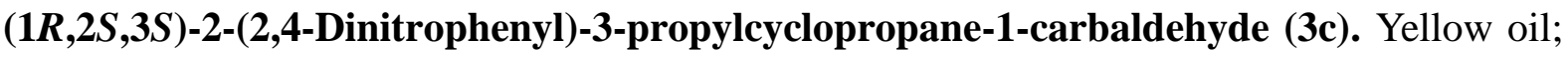
The enantiomeric excess was determined by HPLC using a Chiralpak OD-H column (hexane/ $i$ $\mathrm{PrOH}=80: 20$, flow rate $=1.0 \mathrm{~mL} / \mathrm{min}, \lambda=230 \mathrm{~nm}): \mathrm{t}_{\mathrm{R}}=15.1 \mathrm{~min}, \mathrm{t}_{\mathrm{R}}=16.2 \mathrm{~min} ;[\alpha]_{\mathrm{D}}{ }^{21}=-$ $106.5^{\circ}\left(c 0.9, \mathrm{CHCl}_{3}\right)(S$ catalyst $),[\alpha]_{\mathrm{D}}{ }^{21}=+104.3^{\circ}\left(c\right.$ $\left.0.9, \mathrm{CHCl}_{3}\right)(\boldsymbol{R}$ catalyst $) ;{ }^{1} \mathrm{H} \mathrm{NMR}(400$ $\left.\mathrm{MHz}, \mathrm{CDCl}_{3}\right) \delta=9.44(\mathrm{~d}, J=2.7,1 \mathrm{H}), 8.71(\mathrm{~d}, J=2.2,1 \mathrm{H}), 8.37(\mathrm{dd}, J=8.6,2.1,1 \mathrm{H}), 7.68$ $(\mathrm{d}, J=8.6,1 \mathrm{H}), 2.88(\mathrm{dd}, J=8.1,8.0,1 \mathrm{H}), 2.48(\mathrm{ddd}, J=7.9,4.9,2.91 \mathrm{H}), 2.22-2.14(\mathrm{~m}$, $1 \mathrm{H}), 1.62-1.48(\mathrm{~m}, 4 \mathrm{H}), 0.97(\mathrm{t}, J=7.1,3 \mathrm{H}) ;{ }^{13} \mathrm{C} \mathrm{NMR}\left(101 \mathrm{MHz}, \mathrm{CDCl}_{3}\right) \delta=198.0(\mathrm{CHO})$, $150.1(\mathrm{Cq}), 146.8(\mathrm{Cq}), 139(\mathrm{Cq}), 133.5(\mathrm{CH}), 126.8(\mathrm{CH}), 119.9(\mathrm{CH}), 37.0(\mathrm{CH}), 34.4\left(\mathrm{CH}_{2}\right)$, $33.2(\mathrm{CH}), 29.7(\mathrm{CH}), 22.1\left(\mathrm{CH}_{2}\right), 13.8\left(\mathrm{CH}_{3}\right)$; IR $v_{\max }\left(\mathrm{KBr}, \mathrm{cm}^{-1}\right)$ : 2960, 2930, 2873, 1701, 1604, 1530 (aromatic $\mathrm{NO}_{2}$ ), 1465, 1346 (aromatic $\mathrm{NO}_{2}$ ), 1151, 1067, 1001, 912, 835, 738; HRMS (ESI) calcd for $\mathrm{C}_{13} \mathrm{H}_{14} \mathrm{~N}_{2} \mathrm{O}_{5}[\mathrm{M}+\mathrm{Na}]^{+} 301.0795$, found 301.0792 .

(1S,2S,3S)-2-(2,4-Dinitrophenyl)-3-propylcyclopropane-1-carbaldehyde (4c). Yellow oil; The enantiomeric excess was determined by HPLC using a Chiralpak OD-H column (hexane/i$\mathrm{PrOH}=80: 20$, flow rate $=1.0 \mathrm{~mL} / \mathrm{min}, \lambda=230 \mathrm{~nm}): \mathrm{t}_{\mathrm{R}}=30.6 \mathrm{~min}, \mathrm{t}_{\mathrm{R}}=22.4 \mathrm{~min} ;[\alpha]_{\mathrm{D}}{ }^{21}=$ $+83.4^{\circ}\left(c 0.7, \mathrm{CHCl}_{3}\right)(S$ catalyst $),[\alpha]_{\mathrm{D}}{ }^{21}=-77.8^{\circ}\left(c 0.6, \mathrm{CHCl}_{3}\right)$ ( $\boldsymbol{R}$ catalyst $) ;{ }^{1} \mathrm{H}$ NMR $(400$ $\left.\mathrm{MHz}, \mathrm{CDCl}_{3}\right) \delta=9.65(\mathrm{~d}, J=4.1,1 \mathrm{H}), 8.75(\mathrm{~d}, J=2.2,1 \mathrm{H}), 8.36(\mathrm{dd}, J=8.6,2.2,1 \mathrm{H}), 7.38$ $(\mathrm{d}, J=8.6,1 \mathrm{H}), 3.23(\mathrm{dd}, J=5.9,5.8,1 \mathrm{H}), 2.33(\mathrm{ddd}, J=9.2,4.9,4.2,1 \mathrm{H}), 1.94-1.77(\mathrm{~m}, 2 \mathrm{H})$, $1.69-1.57(\mathrm{~m}, 1 \mathrm{H}), 1.51-1.33(\mathrm{~m}, 2 \mathrm{H}), 0.93(\mathrm{t}, J=7.3,3 \mathrm{H}) ;{ }^{13} \mathrm{C} \mathrm{NMR}\left(101 \mathrm{MHz}, \mathrm{CDCl}_{3}\right) \delta$ $=197.7(\mathrm{CHO}), 150.1(\mathrm{Cq}), 146.5(\mathrm{Cq}), 141.4(\mathrm{Cq}), 129.8(\mathrm{CH}), 127.2(\mathrm{CH}), 120.3(\mathrm{CH}), 36.4$ $(\mathrm{CH}), 33.3(\mathrm{CH}), 29.4\left(\mathrm{CH}_{2}\right), 28.9(\mathrm{CH}), 22.8\left(\mathrm{CH}_{2}\right), 13.7\left(\mathrm{CH}_{3}\right)$; IR v $\max \left(\mathrm{KBr}, \mathrm{cm}^{-1}\right)$ : 2960, 2930, 2873, 1701, 1604, 1530 (aromatic $\mathrm{NO}_{2}$ ), 1465, 1346 (aromatic $\mathrm{NO}_{2}$ ), 1151, 1067, 1001, 912, 835, 738; HRMS (ESI) calcd for $\mathrm{C}_{13} \mathrm{H}_{14} \mathrm{~N}_{2} \mathrm{O}_{5}[\mathrm{M}+\mathrm{Na}]^{+}$301.0795, found 301.0792.

$(1 S, 2 R, 3 R)$-2-(2,4-Dinitrophenyl)-3-propylcyclopropane-1-carbaldehyde (5c). Yellow oil; The enantiomeric excess was determined by HPLC using a Chiralpak OD-H column (hexane/i$\operatorname{PrOH}=80: 20$, flow rate $=1.0 \mathrm{~mL} / \mathrm{min}, \lambda=230 \mathrm{~nm}): \mathrm{t}_{\mathrm{R}}=18.4 \mathrm{~min}, \mathrm{t}_{\mathrm{R}}=31.1 \mathrm{~min},[\alpha]_{\mathrm{D}}{ }^{21}=-$ $116.5^{\circ}\left(c 0.2, \mathrm{CHCl}_{3}\right)(\boldsymbol{S}$ catalyst $),[\alpha]_{\mathrm{D}}{ }^{21}=+130.8^{\circ}\left(c 0.2, \mathrm{CHCl}_{3}\right)$ ( $\boldsymbol{R}$ catalyst $) ;{ }^{1} \mathrm{H} \mathrm{NMR}(400$ $\left.\mathrm{MHz}, \mathrm{CDCl}_{3}\right) \delta=9.43(\mathrm{~d}, J=4.4,1 \mathrm{H}), 8.82(\mathrm{~d}, J=2.1,1 \mathrm{H}), 8.40(\mathrm{dd}, J=8.5,2.1,1 \mathrm{H}), 7.57$ (d, $J=8.5,1 \mathrm{H}), 3.33(\mathrm{dd}, J=9.4,4.6,1 \mathrm{H}), 2.24-2.20(\mathrm{~m}, 1 \mathrm{H}), 2.11-2.04(\mathrm{~m}, 1 \mathrm{H}), 1.39-1.26$ 
$(\mathrm{m}, 4 \mathrm{H}), 0.80(\mathrm{t}, J=7.1,3 \mathrm{H}) ;{ }^{13} \mathrm{C} \mathrm{NMR}\left(101 \mathrm{MHz}, \mathrm{CDCl}_{3}\right) \delta=198.2(\mathrm{CHO}), 150.7(\mathrm{Cq}), 147$ $(\mathrm{Cq}), 138.4(\mathrm{Cq}), 132.8(\mathrm{CH}), 127(\mathrm{CH}), 120.4(\mathrm{CH}), 36.3(\mathrm{CH}), 29.9\left(\mathrm{CH}_{2}\right), 29.5(\mathrm{CH}), 29.1$ $(\mathrm{CH}), 22.0\left(\mathrm{CH}_{2}\right), 13.6\left(\mathrm{CH}_{3}\right)$; IR $v_{\max }\left(\mathrm{KBr}, \mathrm{cm}^{-1}\right): 2960,2930,2873,1701,1604,1530$ (aromatic $\mathrm{NO}_{2}$ ), 1465, 1346 (aromatic $\mathrm{NO}_{2}$ ), 1151, 1067, 1001, 912, 835, 738; HRMS (ESI) calcd for $\mathrm{C}_{13} \mathrm{H}_{14} \mathrm{~N}_{2} \mathrm{O}_{5}[\mathrm{M}+\mathrm{Na}]^{+} 301.0795$, found 301.0796 .

2-(2,4-Dinitrophenyl)-3-heptylcyclopropane-1-carbaldehyde (3d, $4 d$ and 5d): The title compound was synthesized according to general procedure A. The product was purified by column chromatography (hexane/EtOAc $=7: 1$ ), affording the title compound as a yellow oil; Diastereomeric ratios of 3d:4d:5d = 7:1:1; Enantiomeric exceses of 3d:4d:5d = 99:92:54; Total yield of 3c:4c:5c $=99 \%(153 \mathrm{mg})$.

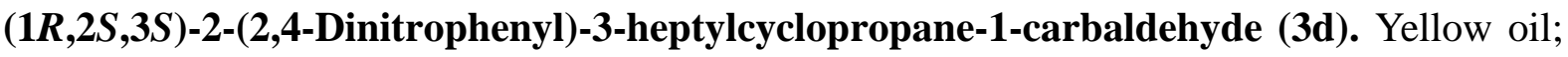
The enantiomeric excess was determined by HPLC using a Chiralpak OD-H column (hexane/ $i$ $\operatorname{PrOH}=80: 20$, flow rate $=1.0 \mathrm{~mL} / \mathrm{min}, \lambda=210 \mathrm{~nm}): \mathrm{t}_{\mathrm{R}}=11.7 \mathrm{~min}, \mathrm{t}_{\mathrm{R}}=12.9 \mathrm{~min} ;[\alpha]_{\mathrm{D}}{ }^{22}=-$ $62.4^{\circ}\left(c 0.9, \mathrm{CHCl}_{3}\right)(S$ catalyst $) .[\alpha]_{\mathrm{D}}{ }^{22}=+77.5^{\circ}\left(c 1.1, \mathrm{CHCl}_{3}\right)$ ( $R$ catalyst $) ;{ }^{1} \mathrm{H} \mathrm{NMR}(400$ $\left.\mathrm{MHz}, \mathrm{CDCl}_{3}\right) \delta=9.41(\mathrm{~d}, J=2.7,1 \mathrm{H}), 8.70(\mathrm{~d}, J=2.1,1 \mathrm{H}), 8.36(\mathrm{dd}, J=8.6,2.3,1 \mathrm{H}), 7.67$ $(\mathrm{d}, J=8.6,1 \mathrm{H}), 2.87(\mathrm{dd}, J=8.2,8.1,1 \mathrm{H}), 2.47(\mathrm{ddd}, J=8.0,4.9,2.8,1 \mathrm{H}), 2.20-2.12(\mathrm{~m}$, $1 \mathrm{H}), 1.62-1.54(\mathrm{~m}, 2 \mathrm{H}), 1.36-1.18(\mathrm{~m}, 10 \mathrm{H}), 0.85(\mathrm{t}, J=6.7,3 \mathrm{H}) ;{ }^{13} \mathrm{C}$ NMR $(101 \mathrm{MHz}$, $\left.\mathrm{CDCl}_{3}\right) \delta=198.1(\mathrm{CHO}), 150.0(\mathrm{Cq}), 146.8(\mathrm{Cq}), 139.1(\mathrm{Cq}), 133.6(\mathrm{CH}), 126.8(\mathrm{CH}), 119.9$ $(\mathrm{CH}), 37.1(\mathrm{CH}), 33.3(\mathrm{CH}), 32.4\left(\mathrm{CH}_{2}\right), 31.7\left(\mathrm{CH}_{2}\right), 29.9(\mathrm{CH}), 29.2\left(\mathrm{CH}_{2}\right), 29.1\left(\mathrm{CH}_{2}\right), 28.9$ $\left(\mathrm{CH}_{2}\right), 22.6\left(\mathrm{CH}_{2}\right), 14.1\left(\mathrm{CH}_{3}\right)$; IR $v_{\max }\left(\mathrm{KBr}, \mathrm{cm}^{-1}\right)$ : 2925, 2854, 1699, 1603, 1530 (aromatic $\mathrm{NO}_{2}$ ), 1465, 1343 (aromatic $\mathrm{NO}_{2}$ ), 1150, 1066, 909, 834, 738, 689, 642, 508; HRMS (ESI) calcd for $\mathrm{C}_{17} \mathrm{H}_{22} \mathrm{~N}_{2} \mathrm{O}_{5}[\mathrm{M}+\mathrm{Na}]^{+}$357.1421, found 357.1421.

(1S,2S,3S)-2-(2,4-Dinitrophenyl)-3-heptylcyclopropane-1-carbaldehyde (4d). Yellow oil; The enantiomeric excess was determined by HPLC using a Chiralpak OD-H column (hexane/i$\operatorname{PrOH}=80: 20$, flow rate $=1.0 \mathrm{~mL} / \mathrm{min}, \lambda=230 \mathrm{~nm}): \mathrm{t}_{\mathrm{R}}=24.4 \mathrm{~min}, \mathrm{t}_{\mathrm{R}}=18.9 \mathrm{~min} ;[\alpha]_{\mathrm{D}}{ }^{22}=$ $+46.8^{\circ}\left(c 0.4, \mathrm{CHCl}_{3}\right)(S$ catalyst $),[\alpha]_{\mathrm{D}}{ }^{22}=-53.1^{\circ}\left(c 1.0, \mathrm{CHCl}_{3}\right)$ ( $R$ catalyst $) ;{ }^{1} \mathrm{H}$ NMR $(400$ $\left.\mathrm{MHz}, \mathrm{CDCl}_{3}\right) \delta=9.64(\mathrm{~d}, J=4.1,1 \mathrm{H}), 8.74(\mathrm{~d}, J=2.3,1 \mathrm{H}), 8.36(\mathrm{dd}, J=8.6,2.3,1 \mathrm{H}), 7.38$ $(\mathrm{d}, J=8.6,1 \mathrm{H}), 3.23(\mathrm{dd}, J=5.9,5.7,1 \mathrm{H}), 2.32(\mathrm{ddd}, J=9.1,4.9,4.4,1 \mathrm{H}), 1.93-1.80(\mathrm{~m}$, 2H), $1.69-1.54(\mathrm{~m}, 1 \mathrm{H}), 1.34-1.18(\mathrm{~m}, 10 \mathrm{H}), 0.85(\mathrm{t}, J=6.8,3 \mathrm{H}) ;{ }^{13} \mathrm{C}$ NMR $(101 \mathrm{MHz}$, $\left.\mathrm{CDCl}_{3}\right) \delta=197.7(\mathrm{CHO}), 150.1(\mathrm{Cq}), 146.5(\mathrm{Cq}), 141.4(\mathrm{Cq}), 129.8(\mathrm{CH}), 127.2(\mathrm{CH}), 120.3$ $(\mathrm{CH}), 36.5(\mathrm{CH}), 33.6(\mathrm{CH}), 31.7\left(\mathrm{CH}_{2}\right), 29.6\left(\mathrm{CH}_{2}\right), 29.2\left(\mathrm{CH}_{2}\right), 29.1\left(\mathrm{CH}_{2}\right), 28.9(\mathrm{CH}), 27.5$ $\left(\mathrm{CH}_{2}\right), 22.6\left(\mathrm{CH}_{2}\right), 14.1\left(\mathrm{CH}_{3}\right)$; IR $v_{\max }\left(\mathrm{KBr}, \mathrm{cm}^{-1}\right)$ : 2925, 2854, 1699, 1603, 1530 (aromatic 
$\mathrm{NO}_{2}$ ), 1465, 1343 (aromatic $\mathrm{NO}_{2}$ ), 1150, 1066, 909, 834, 738, 689, 642, 508; HRMS (ESI) calcd for $\mathrm{C}_{17} \mathrm{H}_{22} \mathrm{~N}_{2} \mathrm{O}_{5}[\mathrm{M}+\mathrm{Na}]^{+} 357.1421$, found 357.1416.

(1S,2R,3R)-2-(2,4-Dinitrophenyl)-3-heptylcyclopropane-1-carbaldehyde (5d). Yellow oil; The enantiomeric excess was determined by HPLC using a Chiralpak OD-H column (hexane/ $i$ $\operatorname{PrOH}=80: 20$, flow rate $=1.0 \mathrm{~mL} / \mathrm{min}, \lambda=230 \mathrm{~nm}): \mathrm{t}_{\mathrm{R}}=15.6 \mathrm{~min}, \mathrm{t}_{\mathrm{R}}=22.7 \mathrm{~min} ;[\alpha]_{\mathrm{D}}{ }^{22}=-$ $71.5^{\circ}\left(c 0.3, \mathrm{CHCl}_{3}\right)(\boldsymbol{S}$ catalyst $),[\alpha]_{\mathrm{D}}{ }^{22}=+132.6^{\circ}\left(c 0.61, \mathrm{CHCl}_{3}\right)(\boldsymbol{R}$ catalyst $) ;{ }^{1} \mathrm{H} \mathrm{NMR}(400$ $\left.\mathrm{MHz}, \mathrm{CDCl}_{3}\right) \delta=9.42(\mathrm{~d}, J=4.4,1 \mathrm{H}), 8.82(\mathrm{~d}, J=2.3,1 \mathrm{H}), 8.40(\mathrm{dd}, J=8.5,2.3,1 \mathrm{H}), 7.57$ $(\mathrm{d}, J=8.5,1 \mathrm{H}), 3.33(\mathrm{dd}, J=9.9,5.4,1 \mathrm{H}), 2.23-2.20(\mathrm{~m}, 1 \mathrm{H}), 2.12-2.00(\mathrm{~m}, 1 \mathrm{H}), 1.32-$ $1.18(\mathrm{~m}, 12 \mathrm{H}), 0.82(\mathrm{t}, J=7.0,3 \mathrm{H}) ;{ }^{13} \mathrm{C} \mathrm{NMR}\left(101 \mathrm{MHz}, \mathrm{CDCl}_{3}\right) \delta=198.3(\mathrm{CHO}), 150.7(\mathrm{Cq})$, $146.9(\mathrm{Cq}), 138.4(\mathrm{Cq}), 132.8(\mathrm{CH}), 127.0(\mathrm{CH}), 120.4(\mathrm{CH}), 36.3(\mathrm{CH}), 33.4(\mathrm{CH}), 31.6\left(\mathrm{CH}_{2}\right)$, $29.7(\mathrm{CH}), 29.2(\mathrm{CH}), 29.0\left(\mathrm{CH}_{2}\right), 28.8\left(\mathrm{CH}_{2}\right), 28.0\left(\mathrm{CH}_{2}\right), 22.5\left(\mathrm{CH}_{2}\right), 14.0\left(\mathrm{CH}_{3}\right)$; IR v $\max$ $\left(\mathrm{KBr}, \mathrm{cm}^{-1}\right)$ : 2925, 2854, 1699, 1603, 1530 (aromatic $\mathrm{NO}_{2}$ ), 1465, 1343 (aromatic $\mathrm{NO}_{2}$ ), 1150, 1066, 909, 834, 738, 689, 642, 508; HRMS (ESI) calcd for $\mathrm{C}_{17} \mathrm{H}_{22} \mathrm{~N}_{2} \mathrm{O}_{5}[\mathrm{M}+\mathrm{Na}]^{+} 357.1421$, found 357.1423 .

2-(But-3-en-1-yl)-3-(2,4-dinitrophenyl)cyclopropane-1-carbaldehyde (3e, 4e and 5e): The title compound was synthesized according to general procedure A. The product was purified by column chromatography (hexane/EtOAc $=7: 1$ ), affording the title compound as a yellow oil; Diastereomeric ratios of 3e:4e:5e $=4: 1: 1$; Enantiomeric exceses of 3e:4e:5e = 99:92:n.d.; Total yield of 3e:4e:5e $=66 \%(89 \mathrm{mg})$.

(1R,2S,3S)-2-(But-3-en-1-yl)-3-(2,4-dinitrophenyl)cyclopropane-1-carbaldehyde

(3e).

Yellow oil; The enantiomeric excess was determined by HPLC using a Chiralpak OD-H column (hexane $/ i-\mathrm{PrOH}=75: 25$, flow rate $1.0 \mathrm{~mL} / \mathrm{min}, \lambda=210 \mathrm{~nm}$ ): $\mathrm{t}_{\mathrm{R}}=14.4 \mathrm{~min}, \mathrm{t}_{\mathrm{R}}=16.4$ $\min ;[\alpha]_{\mathrm{D}}{ }^{23}=-119.1^{\circ}\left(c 1.3, \mathrm{CHCl}_{3}\right)(\boldsymbol{S}$ catalyst $) ;{ }^{1} \mathrm{H} \mathrm{NMR}\left(400 \mathrm{MHz}, \mathrm{CDCl}_{3}\right) \delta=9.44(\mathrm{~d}, J=$ $2.7,1 \mathrm{H}), 8.73(\mathrm{~d}, J=2.4,1 \mathrm{H}), 8.37$ (dd, $J=8.6,2.4,1 \mathrm{H}), 7.68(\mathrm{~d}, J=8.6,1 \mathrm{H}), 5.90-5.74(\mathrm{~m}$, $1 \mathrm{H}), 5.12-4.98(\mathrm{~m}, 2 \mathrm{H}), 2.89(\mathrm{dd}, J=8.2,8.1,1 \mathrm{H}), 2.51(\mathrm{ddd}, J=8.8,5.0,2.7,1 \mathrm{H}), 2.29-$ $2.24(\mathrm{~m}, 2 \mathrm{H}), 2.23-2.16(\mathrm{~m}, 1 \mathrm{H}), 1.80-1.62(\mathrm{~m}, 2 \mathrm{H}) ;{ }^{13} \mathrm{C} \mathrm{NMR}\left(101 \mathrm{MHz}, \mathrm{CDCl}_{3}\right) \delta=197.9$ (CHO), $150.0(\mathrm{Cq}), 146.9(\mathrm{Cq}), 138.8(\mathrm{Cq}), 137.1(\mathrm{CH}), 133.6(\mathrm{CH}), 126.8(\mathrm{CH}), 119.9(\mathrm{CH})$, $116.1\left(\mathrm{CH}_{2}\right), 37.0(\mathrm{CH}), 33.3(\mathrm{CH}), 33.1\left(\mathrm{CH}_{2}\right), 31.6\left(\mathrm{CH}_{2}\right), 29.2(\mathrm{CH})$; IR $v_{\max }\left(\mathrm{KBr}, \mathrm{cm}^{-1}\right)$ : 3079, 2924, 2849, 2211, 2133, 1702, 1640, 1603, 1530 (aromatic $\mathrm{NO}_{2}$ ), 1437, 1346 (aromatic $\mathrm{NO}_{2}$ ), 1150, 1066, 995, 911, 835, 738; HRMS (ESI) calcd for $\mathrm{C}_{14} \mathrm{H}_{14} \mathrm{~N}_{2} \mathrm{O}_{5}[\mathrm{M}+\mathrm{Na}]^{+} 313.0795$, found 313.0797 . 
Yellow oil; The enantiomeric excess was determined by HPLC using a Chiralpak OD-H column (hexane $/ i-\mathrm{PrOH}=75: 25$, flow rate $=1.0 \mathrm{~mL} / \mathrm{min}, \lambda=230 \mathrm{~nm}$ ): $\mathrm{t}_{\mathrm{R}}=25.2 \mathrm{~min}, \mathrm{t}_{\mathrm{R}}=19.6$ $\min ;[\alpha]_{\mathrm{D}}{ }^{23}=+49.0^{\circ}\left(c 0.4, \mathrm{CHCl}_{3}\right)(S$ catalyst $) ;{ }^{1} \mathrm{H} \mathrm{NMR}\left(400 \mathrm{MHz}, \mathrm{CDCl}_{3}\right) \delta=9.68(\mathrm{~d}, J$ $=3.9,1 \mathrm{H}), 8.76(\mathrm{~d}, J=2.4,1 \mathrm{H}), 8.36(\mathrm{dd}, J=8.6,2.4,1 \mathrm{H}), 7.39(\mathrm{~d}, J=8.6,1 \mathrm{H}), 5.77$ (ddt $J$ $=17.0,10.2,6.7,1 \mathrm{H}), 5.08-4.96(\mathrm{~m}, 2 \mathrm{H}), 3.24(\mathrm{dd}, J=6.1,5.91 \mathrm{H}), 2.34(\mathrm{ddd}, J=9.1,5.3$, $3.9,1 \mathrm{H}), 2.22-2.12(\mathrm{~m}, 2 \mathrm{H}), 2.02-1.85(\mathrm{~m}, 2 \mathrm{H}), 1.82-1.72(\mathrm{~m}, 1 \mathrm{H}) ;{ }^{13} \mathrm{C} \mathrm{NMR}(101 \mathrm{MHz}$, $\left.\mathrm{CDCl}_{3}\right) \delta=197.5(\mathrm{CHO}), 150.1(\mathrm{Cq}), 146.6(\mathrm{Cq}), 141.3(\mathrm{Cq}), 137.0(\mathrm{CH}), 129.9(\mathrm{CH}), 127.2$ $(\mathrm{CH}), 120.3(\mathrm{CH}), 116.1\left(\mathrm{CH}_{2}\right), 36.3(\mathrm{CH}), 33.5\left(\mathrm{CH}_{2}\right), 32.8(\mathrm{CH}), 28.9(\mathrm{CH}), 26.5\left(\mathrm{CH}_{2}\right)$; IR $v_{\max }\left(\mathrm{KBr}, \mathrm{cm}^{-1}\right)$ : 3079, 2924, 2849, 2211, 2133, 1702, 1640, 1603, 1530 (aromatic $\mathrm{NO}_{2}$ ), 1437 , 1346 (aromatic $\mathrm{NO}_{2}$ ), 1150, 1066, 995, 911, 835, 738; HRMS (ESI) calcd for $\mathrm{C}_{14} \mathrm{H}_{14} \mathrm{~N}_{2} \mathrm{O}_{5}$ $[\mathrm{M}+\mathrm{Na}]^{+}$313.0795, found 313.0795.

(1R,2S,3R)-2-(But-3-en-1-yl)-3-(2,4-dinitrophenyl)cyclopropane-1-carbaldehyde

(5e).

Yellow oil; ${ }^{1} \mathrm{H}$ NMR $\left(400 \mathrm{MHz}, \mathrm{CDCl}_{3}\right) \delta=9.43(\mathrm{~d}, J=4.3,1 \mathrm{H}), 8.82(\mathrm{~d}, J=2.4,1 \mathrm{H}), 8.40$ (dd, $J=8.5,2.4,1 \mathrm{H}), 7.57(\mathrm{~d}, J=8.5,1 \mathrm{H}), 5.70-5.57(\mathrm{~m}, 1 \mathrm{H}), 4.98-4.85(\mathrm{~m}, 2 \mathrm{H}), 3.33(\mathrm{dd}$, $J=10.0,5.5,1 \mathrm{H}), 2.25-2.22(\mathrm{~m}, 1 \mathrm{H}), 2.12-2.02(\mathrm{~m}, 3 \mathrm{H}), 1.51-1.43(\mathrm{~m}, 1 \mathrm{H}), 0.80-0.68$ $(\mathrm{m}, 1 \mathrm{H}) ;{ }^{13} \mathrm{C}$ NMR $\left(101 \mathrm{MHz}, \mathrm{CDCl}_{3}\right) \delta=198.1(\mathrm{CHO}), 147.0(\mathrm{Cq}), 138.2(\mathrm{Cq}), 136.5(\mathrm{CH})$, $132.8(\mathrm{CH}), 127.0(\mathrm{CH}), 120.4(\mathrm{CH}), 120.3(\mathrm{Cq}), 116.1\left(\mathrm{CH}_{2}\right), 36.2(\mathrm{CH}), 32.9\left(\mathrm{CH}_{2}\right), 29.2$ (CH), $29.1(\mathrm{CH}), 27.3\left(\mathrm{CH}_{2}\right)$; IR $v_{\max }\left(\mathrm{KBr}, \mathrm{cm}^{-1}\right): 3079,2924,2849,2211,2133,1702,1640$, 1603, 1530 (aromatic $\mathrm{NO}_{2}$ ), 1437, 1346 (aromatic $\mathrm{NO}_{2}$ ), 1150, 1066, 995, 911, 835, 738; HRMS (ESI) calcd for $\mathrm{C}_{14} \mathrm{H}_{14} \mathrm{~N}_{2} \mathrm{O}_{5}[\mathrm{M}+\mathrm{Na}]^{+} 313.0795$, found 313.0801 .

Ethyl 2-(2,4-dinitrophenyl)-3-formylcyclopropane-1-carboxylate (3f, 4 f and 5f): The title compound was synthesized according to general procedure A. The product was purified by column chromatography (hexane/EtOAc $=7: 1$ ), affording the title compound as a yellow oil; Diastereomeric ratios of 3f:4f:5f = 1:1:1; Enantiomeric exceses of 3f:4f:5f = 73:40:80; Total yield of 3f:4f:5f $=68 \%(97 \mathrm{mg})$.

Ethyl (1S,2R,3S)-2-(2,4-dinitrophenyl)-3-formylcyclopropane-1-carboxylate (3f). Yellow oil; The enantiomeric excess was determined by HPLC using a Chiralpak OD-H column (hexane $/ i-\mathrm{PrOH}=65: 35$, flow rate $=1.0 \mathrm{~mL} / \mathrm{min}, \lambda=230 \mathrm{~nm}$ ): $\mathrm{t}_{\mathrm{R}}=22.0 \mathrm{~min}, \mathrm{t}_{\mathrm{R}}=42.2 \mathrm{~min}$; $[\alpha]_{\mathrm{D}}{ }^{23}=+1.4^{\circ}\left(c 0.4, \mathrm{CHCl}_{3}\right)(\boldsymbol{S}$ catalyst $),[\alpha]_{\mathrm{D}}{ }^{23}=-0.25^{\circ}\left(\mathrm{c}=1.2, \mathrm{CHCl}_{3}\right)(\boldsymbol{R}$ catalyst $) ;{ }^{1} \mathrm{H}$ $\operatorname{NMR}\left(400 \mathrm{MHz}, \mathrm{CDCl}_{3}\right) \delta=9.60(\mathrm{~d}, J=1.7,1 \mathrm{H}), 8.81(\mathrm{~d}, J=2.3,1 \mathrm{H}), 8.42(\mathrm{dd}, J=8.5,2.3$, $1 \mathrm{H}), 7.70(\mathrm{~d}, J=8.5,1 \mathrm{H}), 4.25(\mathrm{q}, J=7.1,2 \mathrm{H}), 3.51(\mathrm{dd}, J=9.5,6.8,1 \mathrm{H}), 3.21(\mathrm{ddd}, J=9.6$, $4.8,1.7,1 \mathrm{H}), 2.92(\mathrm{dd}, J=6.7,4.8,1 \mathrm{H}), 1.33(\mathrm{t}, J=7.1,1 \mathrm{H}) ;{ }^{13} \mathrm{C} \mathrm{NMR}\left(101 \mathrm{MHz}, \mathrm{CDCl}_{3}\right) \delta$ 
$=195.5(\mathrm{CHO}), 169.6(\mathrm{Cq}), 149.8(\mathrm{Cq}), 147.4(\mathrm{Cq}), 136.2(\mathrm{Cq}), 133.7(\mathrm{CH}), 127.1(\mathrm{CH}), 120.3$

$(\mathrm{CH}), 62.1\left(\mathrm{CH}_{2}\right), 36.1(\mathrm{CH}), 32.4(\mathrm{CH}), 28.6(\mathrm{CH}), 14.2\left(\mathrm{CH}_{3}\right)$; IR $v_{\max }\left(\mathrm{KBr}, \mathrm{cm}^{-1}\right)$ : 2922, 2852, 2248, 2209, 2190, 2182, 2158, 2150, 1727, 1705, 1604, 1530 (aromatic $\mathrm{NO}_{2}$ ), 1466, 1444, 1392, 1344 (aromatic $\mathrm{NO}_{2}$ ), 1286, 1183, 1095, 1050, 1030, 985, 910, 835, 739; HRMS (ESI) calcd for $\mathrm{C}_{13} \mathrm{H}_{12} \mathrm{~N}_{2} \mathrm{O}_{7}[\mathrm{M}+\mathrm{Na}]^{+}$331.0537, found 331.0535.

Ethyl (1S,2R,3R)-2-(2,4-dinitrophenyl)-3-formylcyclopropane-1-carboxylate (4f). Yellow oil; The enantiomeric excess was determined by HPLC using a Chiralpak OD-H column (hexane $/ i-\mathrm{PrOH}=65: 35$, flow rate $=1.0 \mathrm{~mL} / \mathrm{min}, \lambda=230 \mathrm{~nm}$ ): $\mathrm{t}_{\mathrm{R}}=36.8 \mathrm{~min}, \mathrm{t}_{\mathrm{R}}=29.3 \mathrm{~min}$; $[\alpha]_{\mathrm{D}}{ }^{23}=-9.7^{\circ}\left(c 0.5, \mathrm{CHCl}_{3}\right)(\boldsymbol{S}$ catalyst $),[\alpha]_{\mathrm{D}}{ }^{23}=+10.3^{\circ}\left(c 1.4, \mathrm{CHCl}_{3}\right)(\boldsymbol{R}$ catalyst $) ;{ }^{1} \mathrm{H}$ $\operatorname{NMR}\left(400 \mathrm{MHz}, \mathrm{CDCl}_{3}\right) \delta=9.62(\mathrm{~d}, J=5.8,1 \mathrm{H}), 8.90(\mathrm{~d}, J=2.3,1 \mathrm{H}), 8.43(\mathrm{dd}, J=8.5,2.4$, $1 \mathrm{H}), 7.50(\mathrm{~d}, J=8.6,1 \mathrm{H}), 4.31-4.21(\mathrm{~m}, 2 \mathrm{H}), 3.95(\mathrm{dd}, J=6.6,6.6,1 \mathrm{H}), 2.55(\mathrm{dd}, J=9.3$, $6.5,1 \mathrm{H}), 2.40(\mathrm{ddd}, J=9.3,6.6,5.8,1 \mathrm{H}), 1.32(\mathrm{t}, J=7.1,3 \mathrm{H}) ;{ }^{13} \mathrm{C} \mathrm{NMR}\left(101 \mathrm{MHz}, \mathrm{CDCl}_{3}\right)$ $\delta=196.5(\mathrm{CHO}), 168.6(\mathrm{Cq}), 138.3(\mathrm{Cq}), 130.7(\mathrm{CH}), 127.7(\mathrm{CH}), 120.8(\mathrm{CH}), 120.0(\mathrm{Cq})$, $93.1(\mathrm{Cq}), 62.3\left(\mathrm{CH}_{2}\right), 37.0(\mathrm{CH}), 30.4(\mathrm{CH}), 28.5(\mathrm{CH}), 14.1\left(\mathrm{CH}_{3}\right)$; IR $v_{\max }\left(\mathrm{KBr}, \mathrm{cm}^{-1}\right)$ : 2922, 2852, 2248, 2209, 2190, 2182, 2158, 2150, 1727, 1705, 1604, 1530 (aromatic $\mathrm{NO}_{2}$ ), 1466, 1444, 1392, 1344 (aromatic $\mathrm{NO}_{2}$ ), 1286, 1183, 1095, 1050, 1030, 985, 910, 835, 739; HRMS (ESI) calcd for $\mathrm{C}_{13} \mathrm{H}_{12} \mathrm{~N}_{2} \mathrm{O}_{7}[\mathrm{M}+\mathrm{Na}]^{+} 331.0537$, found 331,0539.

Ethyl (1R,2S,3R)-2-(2,4-dinitrophenyl)-3-formylcyclopropane-1-carboxylate (5f). Yellow oil; The enantiomeric excess was determined by HPLC using a Chiralpak OD-H column (hexane $/ \mathrm{PrOH}=65: 35$, flow rate $=1.0 \mathrm{~mL} / \mathrm{min}, \lambda=230 \mathrm{~nm}$ ): $\mathrm{t}_{\mathrm{R}}=20.3 \mathrm{~min}, \mathrm{t}_{\mathrm{R}}=41.8 \mathrm{~min}$; $[\alpha]_{\mathrm{D}}{ }^{23}=-58.0^{\circ}\left(c 0.5, \mathrm{CHCl}_{3}\right)(S$ catalyst $),[\alpha]_{\mathrm{D}}{ }^{23}=+44.1^{\circ}\left(c 0.7, \mathrm{CHCl}_{3}\right)(\boldsymbol{R}$ catalyst $) ;{ }^{1} \mathrm{H}$ $\operatorname{NMR}\left(400 \mathrm{MHz}, \mathrm{CDCl}_{3}\right) \delta=9.68(\mathrm{~d}, J=3.2,1 \mathrm{H}), 8.81(\mathrm{~d}, J=2.3,1 \mathrm{H}), 8.41(\mathrm{dd}, J=8.5,2.3$, $1 \mathrm{H}), 7.67(\mathrm{~d}, J=8.5,1 \mathrm{H}), 4.02-3.93(\mathrm{~m}, 2 \mathrm{H}), 3.50(\mathrm{dd}, J=9.6,6.8,1 \mathrm{H}), 3.02(\mathrm{ddd}, J=6.7$, 4.6, 3.2, 1H), $2.87(\mathrm{dd}, J=9.6,4.6,1 \mathrm{H}), 1.14(\mathrm{t}, J=7.1,3 \mathrm{H}) ;{ }^{13} \mathrm{C} \mathrm{NMR}\left(101 \mathrm{MHz}, \mathrm{CDCl}_{3}\right) \delta$ $=195.9(\mathrm{CHO}), 168.1(\mathrm{Cq}), 150.0(\mathrm{Cq}), 147.3(\mathrm{Cq}), 136.7(\mathrm{Cq}), 133.6(\mathrm{CH}), 127.0(\mathrm{CH}), 120.2$ $(\mathrm{CH}), 62.0\left(\mathrm{CH}_{2}\right), 35.3(\mathrm{CH}), 30.1(\mathrm{CH}), 29.4(\mathrm{CH}), 13.9\left(\mathrm{CH}_{3}\right)$; IR $v_{\max }\left(\mathrm{KBr}, \mathrm{cm}^{-1}\right)$ : 2922, 2852, 2248, 2209, 2190, 2182, 2158, 2150, 1727, 1705, 1604, 1530 (aromatic $\mathrm{NO}_{2}$ ), 1466, 1444, 1392, 1344 (aromatic $\mathrm{NO}_{2}$ ), 1286, 1183, 1095, 1050, 1030, 985, 910, 835, 739; HRMS (ESI) calcd for $\mathrm{C}_{13} \mathrm{H}_{12} \mathrm{~N}_{2} \mathrm{O}_{7}[\mathrm{M}+\mathrm{Na}]^{+}$331.0537, found 331.0531 .

2-(2,4-Dinitrophenyl)-3-phenylcyclopropane-1-carbaldehyde (3g, $4 \mathrm{~g}$ and $5 \mathrm{~g})$ : The title compound was synthesized according to general procedure A. The product was purified by column chromatography (hexane/EtOAc $=5: 1$ ), affording the title compound as a yellow oil; 
Diastereomeric ratios of 3g:4g:5g = 3:2:1; Enantiomeric exceses of 3g:4g:5g = 97:91:96; Total yield of $\mathbf{3 g}: \mathbf{4 g}: \mathbf{5 g}=78 \%(112 \mathrm{mg})$.

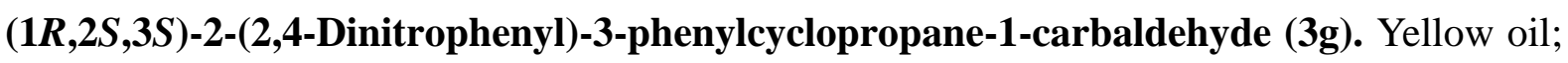
The enantiomeric excess was determined by HPLC using a Chiralpak OD-H column (hexane/ $i$ $\mathrm{PrOH}=70: 30$, flow rate $=1.0 \mathrm{~mL} / \mathrm{min}, \lambda=230 \mathrm{~nm}): \mathrm{t}_{\mathrm{R}}=46.0 \mathrm{~min}, \mathrm{t}_{\mathrm{R}}=52.3 \mathrm{~min} ;[\alpha]_{\mathrm{D}}{ }^{26}=$ $+8.9^{\circ}\left(c 0.7, \mathrm{CHCl}_{3}\right)(\boldsymbol{S}$ catalyst $),[\alpha]_{\mathrm{D}}{ }^{26}=-7.8^{\circ}\left(c 1.4, \mathrm{CHCl}_{3}\right)(\boldsymbol{R}$ catalyst $) ;{ }^{1} \mathrm{H}$ NMR $(400$ $\left.\mathrm{MHz} \mathrm{CDCl}_{3}\right) \delta=9.60(\mathrm{~d}, J=1.9,1 \mathrm{H}), 8.74(\mathrm{~d}, J=2.0,1 \mathrm{H}), 8.39(\mathrm{dd}, J=8.5,2.0,1 \mathrm{H}), 7.80$ $(\mathrm{d}, J=8.6,1 \mathrm{H}), 7.33(\mathrm{t}, J=7.3,2 \mathrm{H}), 7.27(\mathrm{~d}, J=7.1,1 \mathrm{H}), 7.22(\mathrm{~d}, J=8.0,2 \mathrm{H}), 3.40(\mathrm{dd}, J=$ 8.6, 8.0, 1H), $3.27(\mathrm{dd}, J=7.2,5.4,1 \mathrm{H}), 2.93(\mathrm{ddd}, J=7.3,5.2,2.1,1 \mathrm{H}),{ }^{13} \mathrm{C} \mathrm{NMR}(101 \mathrm{MHz}$, $\left.\mathrm{CDCl}_{3}\right) \delta=197(\mathrm{CHO}), 149.9(\mathrm{Cq}), 147.1(\mathrm{Cq}), 138.3(\mathrm{Cq}), 137.1(\mathrm{Cq}), 133.8(\mathrm{CH}), 129.0(2$ $\mathrm{CH}), 127.8(\mathrm{CH}), 127.1(\mathrm{CH}), 126.6(2 \mathrm{CH}), 120.1(\mathrm{CH}), 39.1(\mathrm{CH}), 34.1(\mathrm{CH}), 33.4(\mathrm{CH})$; IR $v_{\max }\left(\mathrm{KBr}, \mathrm{cm}^{-1}\right): 3099,2924,2853,2025,1702,1603,1529$ (aromatic $\left.\mathrm{NO}_{2}\right), 1458,1345$ (aromatic $\mathrm{NO}_{2}$ ), 1151, 1127, 1065, 1031, 1010, 964, 919, 835, 752, 738, 698, 520; HRMS (ESI) calcd for $\mathrm{C}_{16} \mathrm{H}_{12} \mathrm{~N}_{2} \mathrm{O}_{5}[\mathrm{M}+\mathrm{Na}]^{+}$335.0638, found 335.0644.

(1S,2S,3S)-2-(2,4-Dinitrophenyl)-3-phenylcyclopropane-1-carbaldehyde (4g). Yellow oil; The enantiomeric excess was determined by HPLC using a Chiralpak OD-H column (hexane/ $i$ $\mathrm{PrOH}=70: 30$, flow rate $=1.0 \mathrm{~mL} / \mathrm{min}, \lambda=230 \mathrm{~nm}): \mathrm{t}_{\mathrm{R}}=75.3 \mathrm{~min}, \mathrm{t}_{\mathrm{R}}=66.7 \mathrm{~min} ;[\alpha]_{\mathrm{D}}{ }^{26}=-$ $70.8^{\circ}\left(c \quad 0.2, \mathrm{CHCl}_{3}\right)(\boldsymbol{S}$ catalyst $),[\alpha]_{\mathrm{D}}{ }^{26}=+37.8^{\circ}\left(c\right.$ 1.3, $\left.\mathrm{CHCl}_{3}\right)$ ( $\boldsymbol{R}$ catalyst $) ;{ }^{1} \mathrm{H} \mathrm{NMR}(400$ $\left.\mathrm{MHz}, \mathrm{CDCl}_{3}\right) \delta=9.02(\mathrm{~d}, J=5.6,1 \mathrm{H}), 8.82(\mathrm{~d}, J=2.1,1 \mathrm{H}), 8.42(\mathrm{dd}, J=8.6,2.1,1 \mathrm{H}), 7.55$ $(\mathrm{d}, J=8.6,1 \mathrm{H}), 7.42-7.32(\mathrm{~m}, 4 \mathrm{H}), 7.28(\mathrm{~d}, J=6.9,1 \mathrm{H}), 3.97(\mathrm{dd}, J=6.7,5.6,1 \mathrm{H}), 3.23(\mathrm{dd}$, $J=8.8,7.8,1 \mathrm{H}), 2.55(\mathrm{ddd}, J=10.7,9.6,5.4,1 \mathrm{H}) ;{ }^{13} \mathrm{C} \mathrm{NMR}\left(101 \mathrm{MHz}, \mathrm{CDCl}_{3}\right) \delta=197.2$ (CHO), $150.2(\mathrm{Cq}), 146.8(\mathrm{Cq}), 140.5(\mathrm{Cq}), 133.4(\mathrm{Cq}), 130.1(\mathrm{CH}), 128.9(2 \mathrm{CH}), 128.9$ (2 $\mathrm{CH}), 128.1(\mathrm{CH}), 127.5(\mathrm{CH}), 120.5(\mathrm{CH}), 38.3(\mathrm{CH}), 34.9(\mathrm{CH}), 26.2(\mathrm{CH})$; IR $v_{\max }(\mathrm{KBr}$, $\mathrm{cm}^{-1}$ ): 3099, 2924, 2853, 2025, 1702, 1603, 1529 (aromatic $\mathrm{NO}_{2}$ ), 1458,1345 (aromatic $\mathrm{NO}_{2}$ ), 1151, 1127, 1065, 1031, 1010, 964, 919, 835, 752, 738, 698, 520; HRMS (ESI) calcd for $\mathrm{C}_{16} \mathrm{H}_{12} \mathrm{~N}_{2} \mathrm{O}_{5}[\mathrm{M}+\mathrm{Na}]^{+} 335.0638$, found 335.0630.

(1S,2R,3R)-2-(2,4-Dinitrophenyl)-3-phenylcyclopropane-1-carbaldehyde (5g). Yellow oil; The enantiomeric excess was determined by HPLC using a Chiralpak OD-H column (hexane/ $i$ $\operatorname{PrOH}=70: 30$, flow rate $=1.0 \mathrm{~mL} / \mathrm{min}, \lambda=230 \mathrm{~nm}): \mathrm{t}_{\mathrm{R}}=31.0 \mathrm{~min}, \mathrm{t}_{\mathrm{R}}=41.3 \mathrm{~min} ;[\alpha]_{\mathrm{D}}{ }^{26}=-$ $73.9^{\circ}\left(c 0.8, \mathrm{CHCl}_{3}\right)(\boldsymbol{S}$ catalyst $),[\alpha]_{\mathrm{D}}{ }^{26}=+70.8^{\circ}\left(c\right.$ 1.4, $\left.\mathrm{CHCl}_{3}\right)$ ( $\boldsymbol{R}$ catalyst $){ }^{1} \mathrm{H}$ NMR $(400$ $\left.\mathrm{MHz}, \mathrm{CDCl}_{3}\right) \delta=9.69(\mathrm{~d}, J=3.7,1 \mathrm{H}), 8.61(\mathrm{~d}, J=2.1,1 \mathrm{H}), 8.25(\mathrm{dd}, J=8.5,2.1,1 \mathrm{H}), 7.43$ $(\mathrm{d}, J=8.6,1 \mathrm{H}), 7.13-7.06(\mathrm{~m}, 3 \mathrm{H}), 6.82-6.77(\mathrm{~m}, 2 \mathrm{H}), 3.63(\mathrm{dd}, J=10.2,5.7,1 \mathrm{H}), 3.32$ $(\mathrm{dd}, J=10.2,5.1,1 \mathrm{H}), 3.03-2.99(\mathrm{~m}, 1 \mathrm{H}) ;{ }^{13} \mathrm{C} \mathrm{NMR}\left(101 \mathrm{MHz}, \mathrm{CDCl}_{3}\right) \delta=197.6(\mathrm{CHO})$, 
$150.5(\mathrm{Cq}), 146.8(\mathrm{Cq}), 137.5(\mathrm{Cq}), 133.0(\mathrm{CH}), 132.8(\mathrm{Cq}), 128.6(2 \mathrm{CH}), 127.6(3 \mathrm{CH}), 126.7$ $(\mathrm{CH}), 120.2(\mathrm{CH}), 35.4(\mathrm{CH}), 33.1(\mathrm{CH}), 31.4(\mathrm{CH})$; IR $v_{\max }\left(\mathrm{KBr}, \mathrm{cm}^{-1}\right)$ : 3099, 2924, 2853, 2025, 1702, 1603, 1529 (aromatic $\mathrm{NO}_{2}$ ), 1458,1345 (aromatic $\mathrm{NO}_{2}$ ), 1151, 1127, 1065, 1031, 1010, 964, 919, 835, 752, 738, 698, 520; HRMS (ESI) calcd for $\mathrm{C}_{16} \mathrm{H}_{12} \mathrm{~N}_{2} \mathrm{O}_{5}[\mathrm{M}+\mathrm{Na}]^{+}$ 335.0638 , found 335.0641 .

4-(2-(2,4-Dinitrophenyl)-3-formylcyclopropyl)benzonitrile (3h, $4 \mathrm{~h}$ and $5 \mathrm{~h})$ : The title compound was synthesized according to general procedure A. The product was purified by column chromatography (hexane/EtOAc $=6: 1$ to $4: 1$ ), affording the title compound as a yellow oil; Diastereomeric ratios of $\mathbf{3 h}: \mathbf{4 h}: \mathbf{5 h}=2: 1.5: 1$; Enantiomeric exceses of $\mathbf{3 h}: \mathbf{4 h}: \mathbf{5 h}=$ 99:99:n.d.; Total yield of 3h:4h:5h = 80\% (125 mg).

4-((1R,2R,3S)-2-(2,4-Dinitrophenyl)-3-formylcyclopropyl)benzonitrile (3h). Yellow oil; The enantiomeric excess was determined by HPLC using a Chiralpak OD-H column (hexane/i$\operatorname{PrOH}=55: 45$, flow rate $=1.0 \mathrm{~mL} / \mathrm{min}, \lambda=230 \mathrm{~nm}): \mathrm{t}_{\mathrm{R}}=37.1 \mathrm{~min}, \mathrm{t}_{\mathrm{R}}=43.6 \mathrm{~min} ;[\alpha]_{\mathrm{D}}{ }^{23}=-$ $13.9^{\circ}\left(c 0.6, \mathrm{CHCl}_{3}\right)(\boldsymbol{R}$ catalyst $) ;{ }^{1} \mathrm{H} \mathrm{NMR}\left(400 \mathrm{MHz}, \mathrm{CDCl}_{3}\right) \delta=9.68(\mathrm{~d}, J=1.8,1 \mathrm{H}), 8.82$ $(\mathrm{d}, J=2.3,1 \mathrm{H}), 8.44(\mathrm{dd}, J=8.5,2.3,1 \mathrm{H}), 7.79(\mathrm{~d}, J=8.5,1 \mathrm{H}), 7.67(\mathrm{~d}, J=8.2,2 \mathrm{H}), 7.37$ (d, $J=8.3,2 \mathrm{H}), 3.47(\mathrm{dd}, J=8.0,7.6,1 \mathrm{H}), 3.33(\mathrm{dd}, J=7.3,5.3,1 \mathrm{H}), 3.07$ (ddd, $J=9.2,5.2,1.8$, $1 \mathrm{H}) ;{ }^{13} \mathrm{C} \mathrm{NMR}\left(101 \mathrm{MHz}, \mathrm{CDCl}_{3}\right) \delta=196.1(\mathrm{CHO}), 149.9(\mathrm{Cq}), 147.3(\mathrm{Cq}), 142.6(\mathrm{Cq}), 137.2$ $(\mathrm{Cq}), 133.7(\mathrm{CH}), 132.8(\mathrm{CH}), 132.6(\mathrm{CH}), 127.3(\mathrm{CH}), 127.2(\mathrm{CH}), 126.7(\mathrm{CH}), 120.3(\mathrm{CH})$, $118.3(\mathrm{Cq}), 111.7(\mathrm{Cq}), 38.9(\mathrm{CH}), 34.4(\mathrm{CH}), 32.6(\mathrm{CH})$; IR $v_{\max }\left(\mathrm{KBr}, \mathrm{cm}^{-1}\right)$ : 3051, 2921, 1702, 1672, 1628, 1576, 1538, 1499, 1450, 1354, 1327 (aromatic $\mathrm{NO}_{2}$ ), 1134, 1091, 973, 908 ; HRMS (ESI) calcd for $\mathrm{C}_{17} \mathrm{H}_{11} \mathrm{~N}_{3} \mathrm{O}_{5}[\mathrm{M}+\mathrm{Na}]^{+} 360.0591$, found 360.0586.

4-((1R,2R,3R)-2-(2,4-Dinitrophenyl)-3-formylcyclopropyl)benzonitrile $\quad(4 \mathrm{~h}) \quad$ and $\quad 4-$

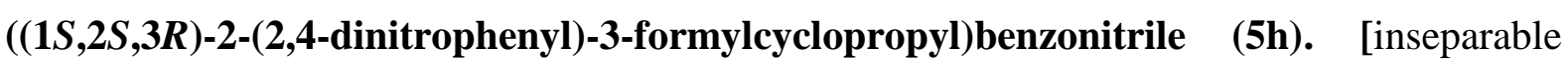
mixture of diastereoisomers]: Yellow oil; The enantiomeric excess of product $\mathbf{4 h}$ was determined by HPLC using a Chiralpak OD-H column (hexane $/ i-\mathrm{PrOH}=65: 35$, flow rate $=$ $1.0 \mathrm{~mL} / \mathrm{min}, \lambda=230 \mathrm{~nm}): \mathrm{t}_{\mathrm{R}}=48.2 \mathrm{~min}, \mathrm{t}_{\mathrm{R}}=45.2 \mathrm{~min} ;{ }^{1} \mathrm{H} \mathrm{NMR}\left(400 \mathrm{MHz}, \mathrm{CDCl}_{3}\right)$ [diastereomer $(\mathbf{4 h})-\mathrm{H}^{\prime}$; diastereomer $\left.(\mathbf{5 h})-\mathrm{H}\right] \delta=9.76\left(\mathrm{~d}, J=3.4,1 \mathrm{H}^{\prime}\right), 9.25(\mathrm{~d}, J=4.2,1 \mathrm{H})$, $8.85(\mathrm{~d}, J=2.3,1 \mathrm{H}), 8.66\left(\mathrm{~d}, J=2.3,1 \mathrm{H}^{\prime}\right), 8.45(\mathrm{dd}, J=8.6,2.4,1 \mathrm{H}), 8.33(\mathrm{dd}, J=8.5,2.4$, $\left.1 \mathrm{H}^{\prime}\right), 7.65(\mathrm{~d}, J=8.4,2 \mathrm{H}), 7.57(\mathrm{~d}, J=8.6,1 \mathrm{H}), 7.51\left(\mathrm{~d}, J=8.6,1 \mathrm{H}^{\prime}\right), 7.42-7.37\left(\mathrm{~m}, 2 \mathrm{H}^{\prime}\right.$ and $2 \mathrm{H}), 6.92\left(\mathrm{~d}, J=8.2,2 \mathrm{H}^{\prime}\right), 4.01(\mathrm{dd}, J=7.2,5.7,1 \mathrm{H}), 3.70\left(\mathrm{dd}, J=10.2,6.0,1 \mathrm{H}^{\prime}\right), 3.35(\mathrm{dd}, J$ $\left.=10.3,5.2,1 \mathrm{H}^{\prime}\right), 3.18(\mathrm{dd}, J=9.3,7.4,1 \mathrm{H}), 3.14\left(\mathrm{dd}, J=5.5,3.4,1 \mathrm{H}^{\prime}\right), 2.77(\mathrm{ddd}, J=9.6,5.5$, 4.2, 1H); ${ }^{13} \mathrm{C}$ NMR $\left(101 \mathrm{MHz}, \mathrm{CDCl}_{3}\right)$ [diastereomer $(\mathbf{4 h})-\mathrm{C}^{\prime}$; diastereomer $\left.(\mathbf{5 h})-\mathrm{C}\right] \delta=$ 
196.7 (C'HO), 195.7 (CHO), 150.3 (C'q), $150.1(\mathrm{Cq}), 147.2\left(\mathrm{C}^{\prime} \mathrm{q}\right), 147.1(\mathrm{Cq}), 139.6(\mathrm{Cq})$, $138.8(\mathrm{Cq}), 138.6\left(\mathrm{C}^{\prime} \mathrm{q}\right), 136.3\left(\mathrm{C}^{\prime} \mathrm{q}\right), 133.0\left(\mathrm{C}^{\prime} \mathrm{H}\right), 132.5(2 \mathrm{CH}), 132.3\left(2 \mathrm{C}^{\prime} \mathrm{H}\right), 130.5(\mathrm{CH})$, 129.8 (2 CH), 128.3 (2 C'H), $127.6(\mathrm{CH}), 127.2\left(\mathrm{C}^{\prime} \mathrm{H}\right), 120.7(\mathrm{CH}), 120.5\left(\mathrm{C}^{\prime} \mathrm{H}\right), 118.3(\mathrm{Cq})$, $118.1\left(\mathrm{C}^{\prime} \mathrm{q}\right), 111.9(\mathrm{Cq}), 111.7\left(\mathrm{C}^{\prime} \mathrm{q}\right), 37.7(\mathrm{CH}), 35.2\left(\mathrm{C}^{\prime} \mathrm{H}\right), 35.1(\mathrm{CH}), 32.8\left(\mathrm{C}^{\prime} \mathrm{H}\right), 32.1\left(\mathrm{C}^{\prime} \mathrm{H}\right)$, $26.7(\mathrm{CH})$; HRMS (ESI) calcd for $\mathrm{C}_{17} \mathrm{H}_{11} \mathrm{~N}_{3} \mathrm{O}_{5}[\mathrm{M}+\mathrm{Na}]^{+} 360.0591$, found 360.0584.

2-(2,4-Dinitrophenyl)-3-(4-nitrophenyl)cyclopropane-1-carbaldehyde (3i, $4 \mathrm{i}$ and $5 \mathrm{i})$ : The title compound was synthesized according to general procedure A. The product was purified by column chromatography (hexane/EtOAc $=6: 1$ ), affording the title compound as a yellow solid; Diastereomeric ratios of 3i:4i:5i = 2:2:1; Enantiomeric exceses of 3i:4i:5i = 98:n.d.:n.d.; Total yield of 3i:4i:5i = 67\% (110.5 mg).

(1R,2S,3S)-2-(2,4-Dinitrophenyl)-3-(4-nitrophenyl)cyclopropane-1-carbaldehyde

(3i).

Yellow solid; The enantiomeric excess was determined by HPLC using a Chiralpak OD-H column (hexane $/ i-\mathrm{PrOH}=45: 55$, flow rate $=1.0 \mathrm{~mL} / \mathrm{min}, \lambda=230 \mathrm{~nm}$ ): $\mathrm{t}_{\mathrm{R}}=42.2 \mathrm{~min}, \mathrm{t}_{\mathrm{R}}=51.3$ $\min ;[\alpha]_{\mathrm{D}}{ }^{23}=+26.3^{\circ}\left(c 0.5, \mathrm{CHCl}_{3}\right)($ S catalyst $) ;{ }^{1} \mathrm{H} \mathrm{NMR}\left(400 \mathrm{MHz}, \mathrm{CDCl}_{3}\right) \delta=9.70(\mathrm{~d}, J=$ $1.8,1 \mathrm{H}), 8.83(\mathrm{~d}, J=2.3,1 \mathrm{H}), 8.46(\mathrm{dd}, J=8.5,2.3,1 \mathrm{H}), 8.24(\mathrm{~d}, J=8.8,2 \mathrm{H}), 7.80(\mathrm{~d}, J=$ $8.6,1 \mathrm{H}), 7.42(\mathrm{~d}, J=8.7,2 \mathrm{H}), 3.51(\mathrm{dd}, J=8.7,7.9,1 \mathrm{H}), 3.38(\mathrm{dd}, J=7.4,5.2,1 \mathrm{H}), 3.12$ (ddd, $J=9.2,5.2,1.9,1 \mathrm{H}) ;{ }^{13} \mathrm{C} \mathrm{NMR}\left(101 \mathrm{MHz}, \mathrm{CDCl}_{3}\right) \delta=196.0(\mathrm{CHO}), 149.9(\mathrm{Cq}), 147.4(\mathrm{Cq})$, 147.4 (Cq), 144.6 (Cq), 137.1 (Cq), 133.7 (CH), 127.4 (2 CH), 127.2 (CH), $124.3(2 \mathrm{CH}), 120.3$ $(\mathrm{CH}), 39.0(\mathrm{CH}), 34.6(\mathrm{CH}), 32.4(\mathrm{CH})$; IR $v_{\max }\left(\mathrm{KBr}, \mathrm{cm}^{-1}\right): 2922,2852,2159,2054,1703$, 1602, 1518 (aromatic $\mathrm{NO}_{2}$ ), 1345 (aromatic $\mathrm{NO}_{2}$ ), 1151, 1111, 1065, 1012, 960, 919, 854, 835, 749, 694; HRMS (ESI) calcd for $\mathrm{C}_{16} \mathrm{H}_{11} \mathrm{~N}_{3} \mathrm{O}_{7}[\mathrm{M}+\mathrm{Na}]^{+} 380.0489$, found 380.0480.

(1S,2S,3S)-2-(2,4-Dinitrophenyl)-3-(4-nitrophenyl)cyclopropane-1-carbaldehyde (4i) and (1S,2R,3R)-2-(2,4-dinitrophenyl)-3-(4-nitrophenyl)cyclopropane-1-carbaldehyde

(5i). [inseparable mixture of diastereoisomers]: Yellow oil; ${ }^{1} \mathrm{H}$ NMR (400 $\mathrm{MHz}, \mathrm{CDCl}_{3}$ ) [diastereomer $(\mathbf{4 i})-\mathrm{H}^{\prime}$; diastereomer $\left.(\mathbf{5 i})-\mathrm{H}\right] \delta=9.79\left(\mathrm{~d}, J=3.3,1 \mathrm{H}^{\prime}\right), 9.29(\mathrm{~d}, J=4.0,1 \mathrm{H})$, $8.86(\mathrm{~d}, J=2.3,1 \mathrm{H}), 8.65\left(\mathrm{~d}, J=2.3,1 \mathrm{H}^{\prime}\right), 8.46(\mathrm{dd}, J=8.6,2.4,1 \mathrm{H}), 8.34(\mathrm{dd}, J=8.5,2.3$, $\left.1 \mathrm{H}^{\prime}\right), 8.20(\mathrm{~d}, J=8.8,2 \mathrm{H}), 7.96\left(\mathrm{~d}, J=8.8,2 \mathrm{H}^{\prime}\right), 7.58(\mathrm{t}, J=9.1 \mathrm{~Hz}, 3 \mathrm{H}), 7.54(\mathrm{~d}, J=8.6 \mathrm{~Hz}$, $\left.1 \mathrm{H}^{\prime}\right), 6.98\left(\mathrm{~d}, J=8.7,2 \mathrm{H}^{\prime}\right), 4.04(\mathrm{dd}, J=7.3,5.7,1 \mathrm{H}), 3.72\left(\mathrm{dd}, J=10.2,6.0,1 \mathrm{H}^{\prime}\right), 3.40(\mathrm{dd}$, $\left.J=10.3,5.2,1 \mathrm{H}^{\prime}\right), 3.22(\mathrm{dd}, J=9.4,7.5,1 \mathrm{H}), 3.14\left(\mathrm{ddd}, J=8.8,5.5,3.3,1 \mathrm{H}^{\prime}\right), 2.82(\mathrm{ddd}, J=$ 9.5, 5.5, 4.0, 1H); $\left.{ }^{13} \mathrm{C} \mathrm{NMR} \mathrm{(101} \mathrm{MHz}, \mathrm{CDCl}_{3}\right)$ [diastereomer (4i) - $\mathrm{C}^{\prime}$; diastereomer $(\mathbf{5 i})-\mathrm{C}$ ] $\delta=196.6\left(\mathrm{C}^{\prime} \mathrm{HO}\right), 195.5(\mathrm{CHO}), 150.3(\mathrm{Cq}), 150.1(\mathrm{Cq}), 147.5\left(\mathrm{C}^{\prime} \mathrm{q}\right), 147.3\left(\mathrm{C}^{\prime} \mathrm{q}\right), 147.2\left(\mathrm{C}^{\prime} \mathrm{q}\right)$, 
$140.8(\mathrm{Cq}), 140.7\left(\mathrm{C}^{\prime} \mathrm{q}\right), 139.5(\mathrm{Cq}), 136.2\left(\mathrm{C}^{\prime} \mathrm{q}\right), 133.0\left(\mathrm{C}^{\prime} \mathrm{H}\right), 130.5\left(\mathrm{C}^{\prime} \mathrm{H}\right), 129.9(2 \mathrm{CH}), 128.4$ (2 C'H), $127.7(\mathrm{CH}), 127.2\left(\mathrm{C}^{\prime} \mathrm{H}\right), 126.4(\mathrm{Cq}), 124.0$ (2 CH), 123.8 (2 C'H), $120.7(\mathrm{CH}), 120.5$ $(\mathrm{CH}), 37.7(\mathrm{CH}), 35.4\left(\mathrm{C}^{\prime} \mathrm{H}\right), 34 .(\mathrm{CH}), 32.7\left(\mathrm{C}^{\prime} \mathrm{H}\right), 32.3\left(\mathrm{C}^{\prime} \mathrm{H}\right), 27.0(\mathrm{CH})$; IR $v_{\max }\left(\mathrm{KBr}, \mathrm{cm}^{-1}\right)$ : 2922, 2852, 2159, 2054, 1703, 1602, 1518 (aromatic $\mathrm{NO}_{2}$ ), 1345 (aromatic $\mathrm{NO}_{2}$ ), 1151, 1111, 1065, 1012, 960, 919, 854, 835, 749, 694; HRMS (ESI) calcd for $\mathrm{C}_{16} \mathrm{H}_{11} \mathrm{~N}_{3} \mathrm{O}_{7}[\mathrm{M}+\mathrm{Na}]^{+}$ 380.0489 , found 380.0486 .

\section{2-(2,4-Dinitrophenyl)-3-(4-fluorophenyl)cyclopropane-1-carbaldehyde (3j, $4 \mathbf{j}$ and $5 \mathbf{j})$ :}

The title compound was synthesized according to general procedure A. The product was purified by column chromatography (hexane/EtOAc $=7: 1$ ), affording the title compound as an

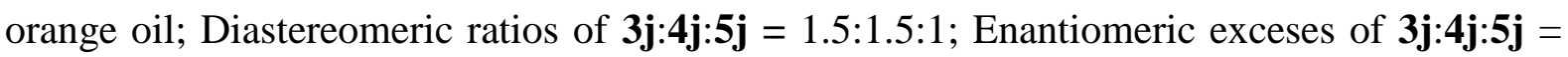
98:91:90; Total yield of $\mathbf{3 j}: \mathbf{4 j}: \mathbf{5 j}=78 \%(119 \mathrm{mg})$.

(1R,2S,3S)-2-(2,4-Dinitrophenyl)-3-(4-fluorophenyl)cyclopropane-1-carbaldehyde (3j).

Yellow oil; The enantiomeric excess was determined by HPLC using a Chiralpak AD-H column (hexane $/ i-\mathrm{PrOH}=85: 15$, flow rate $=1.0 \mathrm{~mL} / \mathrm{min}, \lambda=245 \mathrm{~nm}$ ): $\mathrm{t}_{\mathrm{R}}=25.2 \mathrm{~min}, \mathrm{t}_{\mathrm{R}}=33.0$ $\min ;[\alpha]_{\mathrm{D}}{ }^{20}=+7.3^{\circ}\left(c 1.0, \mathrm{CHCl}_{3}\right)(S$ catalyst $) ;{ }^{1} \mathrm{H} \mathrm{NMR}\left(500 \mathrm{MHz}, \mathrm{CDCl}_{3}\right) \delta=9.67(\mathrm{~d}, J=$ $2,1 \mathrm{H}), 8.82(\mathrm{~d}, J=2,1 \mathrm{H}), 8.46(\mathrm{dd}, J=8.5,2.5,1 \mathrm{H}), 7.85(\mathrm{~d}, J=8.5,1 \mathrm{H}), 7.25(\mathrm{~m}, 2 \mathrm{H}), 7.08$ $(\mathrm{m}, 2 \mathrm{H}), 3.43(\mathrm{t}, J=8.3,1 \mathrm{H}), 3.33(\mathrm{dd}, J=7.5,5.5,1 \mathrm{H}), 3.00(\mathrm{ddd}, J=9.3,5.3,2.3,1 \mathrm{H}) ;{ }^{13} \mathrm{C}$ NMR (125 MHz, $\left.\mathrm{CDCl}_{3}\right) \delta=197.0(\mathrm{CHO}), 162.1$ (d, J=247.7Hz, Cq), $149.9(\mathrm{Cq}), 147.2(\mathrm{Cq})$, $138.2(\mathrm{Cq}), 133.9(\mathrm{CH}), 132.9(\mathrm{CH}), 128.5$ (d, J=8.1Hz, CH), $127.2(\mathrm{CH}), 120.3(\mathrm{CH}), 116.1$ (d, J=21.7Hz, CH), $39.1(\mathrm{CH}), 34.2(\mathrm{CH}), 32.7(\mathrm{CH})$; IR $v_{\max }\left(\mathrm{KBr}, \mathrm{cm}^{-1}\right)$ : 2957, 2921, 2855, 2803, 2368, 2356, 1703, 1605, 1535 (aromatic $\mathrm{NO}_{2}$ ), 1515, 1349 (aromatic $\mathrm{NO}_{2}$ ), 1229, 1161, 912, 836, 822, 737, 670, 636; HRMS (ESI) calcd for $\mathrm{C}_{16} \mathrm{H}_{11} \mathrm{FN}_{2} \mathrm{O}_{5}[\mathrm{M}+\mathrm{H}]^{+} 331.0725$, found 331.0726 .

(1S,2S,3S)-2-(2,4-Dinitrophenyl)-3-(4-fluorophenyl)cyclopropane-1-carbaldehyde (4j).

Yellow oil; The enantiomeric excess was determined by HPLC using a Chiralpak AD-H column (hexane $/ i-\mathrm{PrOH}=85: 15$, flow rate $=1.0 \mathrm{~mL} / \mathrm{min}, \lambda=245 \mathrm{~nm}$ ): $\mathrm{t}_{\mathrm{R}}=15.4 \mathrm{~min}, \mathrm{t}_{\mathrm{R}}=20.6$ $\min ;[\alpha]_{\mathrm{D}}{ }^{20}=-49.4^{\circ}\left(c 0.56, \mathrm{CHCl}_{3}\right)(S$ catalyst $) ;{ }^{1} \mathrm{H} \mathrm{NMR}\left(500 \mathrm{MHz}, \mathrm{CDCl}_{3}\right) \delta=9.12(\mathrm{~d}, J$ $=5,1 \mathrm{H}), 8.85(\mathrm{~d}, J=2.5,1 \mathrm{H}), 8.45(\mathrm{dd}, J=8.7,2.3,1 \mathrm{H}), 7.57(\mathrm{~d}, J=8.5,1 \mathrm{H}), 7.39(\mathrm{dd}, J=$ $8.5,5.5,2 \mathrm{H}), 7.07(\mathrm{t}, J=8.7,2 \mathrm{H}), 3.96(\mathrm{dd}, J=6.5,5.5,1 \mathrm{H}), 3.20(\mathrm{t}, J=8.3,1 \mathrm{H}), 2.62(\mathrm{dt}, J$ $=9.5,5,1 \mathrm{H}) ;{ }^{13} \mathrm{C} \mathrm{NMR}\left(125 \mathrm{MHz}, \mathrm{CDCl}_{3}\right) \delta=196.9(\mathrm{CHO}), 162.1(\mathrm{~d}, \mathrm{~J}=247.5 \mathrm{~Hz}, \mathrm{Cq}), 150.1$ (Cq), $146.9(\mathrm{Cq}), 140.3(\mathrm{Cq}), 130.6$ (d, J=8.2Hz, CH), $130.2(\mathrm{CH}), 129.4$ (d, J=8.1Hz, CH), $127.5(\mathrm{CH}), 120.6(\mathrm{CH}), 115.8(\mathrm{~d}, \mathrm{~J}=21.7 \mathrm{~Hz}, \mathrm{CH}), 37.9(\mathrm{CH}), 34.4(\mathrm{CH}), 26.6(\mathrm{CH})$; IR $v_{\max }$ 
$\left(\mathrm{KBr}, \mathrm{cm}^{-1}\right):$ 2957, 2921, 2855, 2803, 2368, 2356, 1703, 1605, 1535 (aromatic $\mathrm{NO}_{2}$ ), 1515, 1349 (aromatic $\mathrm{NO}_{2}$ ), 1229, 1161, 912, 836, 822, 737, 670, 636; HRMS (ESI) calcd for $\mathrm{C}_{16} \mathrm{H}_{11} \mathrm{FN}_{2} \mathrm{O}_{5}[\mathrm{M}+\mathrm{H}]^{+} 331.0725$, found 331.0726.

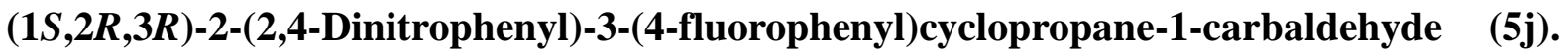
Yellow oil; The enantiomeric excess was determined by HPLC using a Chiralpak AD-H column (hexane $/ i-\mathrm{PrOH}=90: 10$, flow rate $=1.0 \mathrm{~mL} / \mathrm{min}, \lambda=245 \mathrm{~nm}): \mathrm{t}_{\mathrm{R}}=27.8 \mathrm{~min}, \mathrm{t}_{\mathrm{R}}=36.8$; $[\alpha]_{\mathrm{D}}{ }^{20}=-52.8^{\circ}\left(c 0.51, \mathrm{CHCl}_{3}\right)(\boldsymbol{S}$ catalyst $) ;{ }^{1} \mathrm{H} \mathrm{NMR}\left(500 \mathrm{MHz}, \mathrm{CDCl}_{3}\right) \delta=9.72(\mathrm{~d}, J=3.5$, $1 \mathrm{H}), 8.67(\mathrm{~d}, J=2.5,1 \mathrm{H}), 8.30(\mathrm{dd}, J=8.7,2.3,1 \mathrm{H}), 7.45(\mathrm{~d}, J=8.5,1 \mathrm{H}), 6.84-6.78(\mathrm{~m}, 4 \mathrm{H})$, $3.63(\mathrm{dd}, J=10.3,5.8,1 \mathrm{H}), 3.33(\mathrm{dd}, J=10.5,5,1 \mathrm{H}), 3.01(\mathrm{~m}, 1 \mathrm{H}) ;{ }^{13} \mathrm{C}$ NMR $(125 \mathrm{MHz}$, $\left.\mathrm{CDCl}_{3}\right) \delta=197.6(\mathrm{CHO}), 162.1(\mathrm{~d}, \mathrm{~J}=247.7 \mathrm{~Hz}, \mathrm{Cq}), 150.5(\mathrm{Cq}), 147.0(\mathrm{Cq}), 137.3(\mathrm{Cq}), 133.0$ $(\mathrm{CH}), 129.4(\mathrm{CH}), 128.6(\mathrm{CH}), 126.9(\mathrm{CH}), 120.4(\mathrm{CH}), 115.8(\mathrm{~d}, \mathrm{~J}=21.7 \mathrm{~Hz}, \mathrm{CH}), 35.5(\mathrm{CH})$, $32.5(\mathrm{CH}), 31.5(\mathrm{CH})$; IR $v_{\max }\left(\mathrm{KBr}, \mathrm{cm}^{-1}\right): 2957,2921,2855,2803,2368,2356,1703,1605$, 1535 (aromatic $\mathrm{NO}_{2}$ ), 1515, 1349 (aromatic $\mathrm{NO}_{2}$ ), 1229, 1161, 912, 836, 822, 737, 670, 636; HRMS (ESI) calcd for $\mathrm{C}_{16} \mathrm{H}_{11} \mathrm{FN}_{2} \mathrm{O}_{5}[\mathrm{M}+\mathrm{H}]^{+} 331.0725$, found 331.0728 .

\section{2-(4-Chlorophenyl)-3-(2,4-dinitrophenyl)cyclopropane-1-carbaldehyde (3k, 4k and 5k):}

The title compound was synthesized according to general procedure A. The product was purified by column chromatography (hexane/EtOAc $=5: 1$ ), affording the title compound as a yellow oil; Diastereomeric ratios of $3 \mathbf{k}: 4 \mathbf{k}: \mathbf{5 k}=2.5: 1.5: 1$; Enantiomeric exceses of $3 \mathbf{k}: 4 \mathbf{k}: 5 \mathbf{k}$ = 99:94:97; Total yield of 3k:4k:5k = 86\% (137 mg).

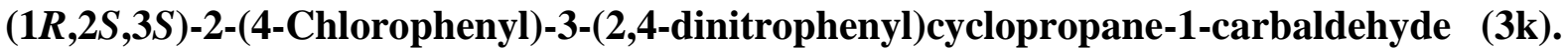
Yellow oil; The enantiomeric excess was determined by HPLC using a Chiralpak OD-H column (hexane $/ i-\mathrm{PrOH}=55: 45$, flow rate $=1.0 \mathrm{~mL} / \mathrm{min}, \lambda=230 \mathrm{~nm}$ ): $\mathrm{t}_{\mathrm{R}}=24.4 \mathrm{~min}, \mathrm{t}_{\mathrm{R}}=31.8$ $\min ;[\alpha]_{\mathrm{D}}{ }^{23}=+21.1^{\circ}\left(c 0.7, \mathrm{CHCl}_{3}\right)($ S catalyst $) ;{ }^{1} \mathrm{H} \mathrm{NMR}\left(400 \mathrm{MHz}, \mathrm{CDCl}_{3}\right) \delta=9.64(\mathrm{~d}, J=$ $2.0,1 \mathrm{H}), 8.79(\mathrm{~d}, J=2.3,1 \mathrm{H}), 8.43(\mathrm{dd}, J=8.5,2.3,1 \mathrm{H}), 7.81(\mathrm{~d}, J=8.5,1 \mathrm{H}), 7.33(\mathrm{~d}, J=$ $8.5,2 \mathrm{H}), 7.19(\mathrm{~d}, J=8.4,2 \mathrm{H}), 3.40(\mathrm{dd}, J=8.5,8.2,1 \mathrm{H}), 3.28(\mathrm{dd}, J=7.4,5.2,1 \mathrm{H}), 2.98$ (ddd, $J=9.1,5.1,2.1,1 \mathrm{H}) ;{ }^{13} \mathrm{C} \mathrm{NMR}\left(101 \mathrm{MHz}, \mathrm{CDCl}_{3}\right) \delta=196.6(\mathrm{CHO}), 149.9(\mathrm{Cq}), 147.2(\mathrm{Cq})$, $137.9(\mathrm{Cq}), 135.6(\mathrm{Cq}), 133.7(\mathrm{CH}), 133.7(\mathrm{Cq}), 129.2(2 \mathrm{CH}), 127.9(2 \mathrm{CH}), 127.1(\mathrm{CH}), 120.1$ $(\mathrm{CH}), 38.9(\mathrm{CH}), 34.1(\mathrm{CH}), 32.5(\mathrm{CH})$; IR $v_{\max }\left(\mathrm{KBr}, \mathrm{cm}^{-1}\right)$ : 3099, 2925, 2854, 1702, 1603, 1529 (aromatic $\mathrm{NO}_{2}$ ), 1496, 1435, 1397, 1345 (aromatic $\mathrm{NO}_{2}$ ), 1214, 1151, 1126, 1092, 1066, $1038,1013,964,918,835,811,759,739,668$; HRMS (ESI) calcd for $\mathrm{C}_{16} \mathrm{H}_{11} \mathrm{ClN}_{2} \mathrm{O}_{5}[\mathrm{M}+\mathrm{Na}]^{+}$ 369.0249 , found 369.0247 .

(1S,2S,3S)-2-(4-Chlorophenyl)-3-(2,4-dinitrophenyl)cyclopropane-1-carbaldehyde (4k). 
Yellow oil; The enantiomeric excess was determined by HPLC using a Chiralpak OD-H column (hexane $/ i-\mathrm{PrOH}=55: 45$, flow rate $=1.0 \mathrm{~mL} / \mathrm{min}, \lambda=230 \mathrm{~nm}$ ): $\mathrm{t}_{\mathrm{R}}=43.8 \mathrm{~min}, \mathrm{t}_{\mathrm{R}}=39.4$ $\min ;[\alpha]_{\mathrm{D}}^{22}=-75.7^{\circ}\left(c 0.9, \mathrm{CHCl}_{3}\right)(\boldsymbol{S}$ catalyst $),[\alpha]_{\mathrm{D}}^{22}=+72.2^{\circ}\left(c 0.8, \mathrm{CHCl}_{3}\right)(\boldsymbol{R}$ catalyst $)$; ${ }^{1} \mathrm{H}$ NMR $\left(400 \mathrm{MHz}, \mathrm{CDCl}_{3}\right) \delta=9.10(\mathrm{~d}, J=5.0,1 \mathrm{H}), 8.81(\mathrm{~d}, J=2.3,1 \mathrm{H}), 8.42(\mathrm{dd}, J=8.6$, $2.3,1 \mathrm{H}), 7.54(\mathrm{~d}, J=8.6,1 \mathrm{H}), 7.35-7.27(\mathrm{~m}, 4 \mathrm{H}), 3.93(\mathrm{dd}, J=7.0,5.6,1 \mathrm{H}), 3.15(\mathrm{dd}, J=$ 9.4, 7.2, 1H), $2.61(\mathrm{ddd}, J=10.0,9.8,5.2,1 \mathrm{H}) ;{ }^{13} \mathrm{C} \mathrm{NMR}\left(101 \mathrm{MHz}, \mathrm{CDCl}_{3}\right) \delta=196.6(\mathrm{CHO})$, $150.1(\mathrm{Cq}), 146.9(\mathrm{Cq}), 140.1(\mathrm{Cq}), 134(\mathrm{Cq}), 131.9(\mathrm{Cq}), 130.3(3 \mathrm{CH}), 129.1(2 \mathrm{CH}), 127.5$ $(\mathrm{CH}), 120.5(\mathrm{CH}), 37.9(\mathrm{CH}), 34.5(\mathrm{CH}), 26.5(\mathrm{CH})$; IR $v_{\max }\left(\mathrm{KBr}, \mathrm{cm}^{-1}\right)$ : 3099, 2925, 2854, 1702, 1603, 1529 (aromatic $\mathrm{NO}_{2}$ ), 1496, 1435, 1397, 1345 (aromatic $\mathrm{NO}_{2}$ ), 1214, 1151, 1126, 1092, 1066, 1038, 1013, 964, 918, 835, 811, 759, 739, 668; HRMS (ESI) calcd for $\mathrm{C}_{16} \mathrm{H}_{11} \mathrm{ClN}_{2} \mathrm{O}_{5}[\mathrm{M}+\mathrm{Na}]^{+}$369.0249, found 369.0240.

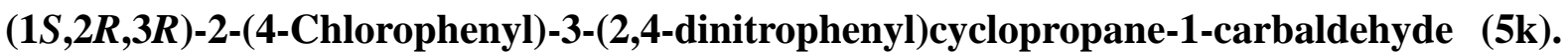
Yellow oil; The enantiomeric excess was determined by HPLC using a Chiralpak OD-H column (hexane $/ i-\mathrm{PrOH}=60: 40$, flow rate $=1.0 \mathrm{~mL} / \mathrm{min}, \lambda=230 \mathrm{~nm}$ ): $\mathrm{t}_{\mathrm{R}}=29.1 \mathrm{~min}, \mathrm{t}_{\mathrm{R}}=32.6$ $\min ;[\alpha]_{\mathrm{D}}^{22}=-56.1^{\circ}\left(c 0.6, \mathrm{CHCl}_{3}\right)(\boldsymbol{S}$ catalyst $),[\alpha]_{\mathrm{D}}^{22}=+56.2^{\circ}\left(c 0.7, \mathrm{CHCl}_{3}\right)(\boldsymbol{R}$ catalyst $) ;$ ${ }^{1} \mathrm{H}$ NMR $\left(400 \mathrm{MHz}, \mathrm{CDCl}_{3}\right) \delta=9.71(\mathrm{~d}, J=3.6,1 \mathrm{H}), 8.66(\mathrm{~d}, J=2.3,1 \mathrm{H}), 8.29(\mathrm{dd}, J=8.5$, $2.3,1 \mathrm{H}), 7.42(\mathrm{~d}, J=8.6,1 \mathrm{H}), 7.08(\mathrm{~d}, J=8.5,2 \mathrm{H}), 6.75(\mathrm{~d}, J=8.5,2 \mathrm{H}), 3.62(\mathrm{dd}, J=10.2$, $5.8,1 \mathrm{H}), 3.29(\mathrm{dd}, J=10.2,5.2,1 \mathrm{H}), 3.01-2.97(\mathrm{~m}, 1 \mathrm{H}) ;{ }^{13} \mathrm{C} \mathrm{NMR}\left(101 \mathrm{MHz}, \mathrm{CDCl}_{3}\right) \delta=$ $197.2(\mathrm{CHO}), 150.4(\mathrm{Cq}), 147(\mathrm{Cq}), 137(\mathrm{Cq}), 133.7(\mathrm{Cq}), 132.8(\mathrm{CH}), 131.4(\mathrm{Cq}), 128.9$ (2 CH), $128.9(2 \mathrm{CH}), 126.7(\mathrm{CH}), 120.4(\mathrm{CH}), 35.2(\mathrm{CH}), 32.5(\mathrm{CH}), 31.4(\mathrm{CH})$; IR $v_{\max }(\mathrm{KBr}$, $\mathrm{cm}^{-1}$ ): 3099, 2925, 2854, 1702, 1603, 1529 (aromatic $\mathrm{NO}_{2}$ ), 1496, 1435, 1397, 1345 (aromatic $\left.\mathrm{NO}_{2}\right), 1214,1151,1126,1092,1066,1038,1013,964,918,835,811,759,739,668$; HRMS (ESI) calcd for $\mathrm{C}_{16} \mathrm{H}_{11} \mathrm{ClN}_{2} \mathrm{O}_{5}[\mathrm{M}+\mathrm{Na}]^{+} 369.0249$, found 369.0249.

\section{2-(4-Bromophenyl)-3-(2,4-dinitrophenyl)cyclopropane-1-carbaldehyde (31, 41 and 5l):}

The title compound was synthesized according to general procedure A. The product was purified by column chromatography (hexane/EtOAc $=5: 1$ ), affording the title compound as a yellow solid; Diastereomeric ratios of 31:4l:5l $=2: 2: 1$; Enantiomeric exceses of 31:4l:5l = 99:95:98; Total yield of 31:41:5l = 71\% (129 mg).

(1S,2R,3R)-2-(4-Bromophenyl)-3-(2,4-dinitrophenyl)cyclopropane-1-carbaldehyde (3I). Yellow solid; M.p.:117 ${ }^{\circ} \mathrm{C}$; The enantiomeric excess was determined by HPLC using a Chiralpak OD-H column (hexane $/ i-\mathrm{PrOH}=70: 30$, flow rate $=1.0 \mathrm{~mL} / \mathrm{min}, \lambda=230 \mathrm{~nm}$ ): $\mathrm{t}_{\mathrm{R}}=$ $40.6 \mathrm{~min}, \mathrm{t}_{\mathrm{R}}=56.2 \mathrm{~min} ;[\alpha]_{\mathrm{D}}{ }^{22}=-2.4^{\circ}\left(c 0.5, \mathrm{CHCl}_{3}\right)(\boldsymbol{R}$ catalyst $) ;{ }^{1} \mathrm{H} \mathrm{NMR}\left(400 \mathrm{MHz}, \mathrm{CDCl}_{3}\right)$ 
$\delta=9.65(\mathrm{~d}, J=2.0,1 \mathrm{H}), 8.80(\mathrm{~d}, J=2.3,1 \mathrm{H}), 8.44(\mathrm{dd}, J=8.5,2.3,1 \mathrm{H}), 7.81(\mathrm{~d}, J=8.6,1 \mathrm{H})$, $7.49(\mathrm{~d}, J=8.5,2 \mathrm{H}), 7.13(\mathrm{~d}, J=8.4,2 \mathrm{H}), 3.41(\mathrm{dd}, J=8.5,8.2,1 \mathrm{H}), 3.26(\mathrm{dd}, J=7.5,5.2$, 1H), $2.99(\mathrm{ddd}, J=9.1,5.1,2.1,1 \mathrm{H}) ;{ }^{13} \mathrm{C} \mathrm{NMR}\left(101 \mathrm{MHz}, \mathrm{CDCl}_{3}\right) \delta=196.7(\mathrm{CHO}), 149.9$ $(\mathrm{Cq}), 147.2(\mathrm{Cq}), 137.9(\mathrm{Cq}), 136.1(\mathrm{Cq}), 133.7(\mathrm{CH}), 132.1(2 \mathrm{CH}), 128.3(2 \mathrm{CH}), 127.1(\mathrm{CH})$, $121.6(\mathrm{Cq}), 120.2(\mathrm{CH}), 38.9(\mathrm{CH}), 34.1(\mathrm{CH}), 32.6(\mathrm{CH})$; IR $v_{\max }\left(\mathrm{KBr}, \mathrm{cm}^{-1}\right)$ : 2998, 2925, 1712, 1673, 1622, 1576, 1538, 1499, 1450, 1354, 1327 (aromatic $\mathrm{NO}_{2}$ ), 1134, 1091, 973, 908; HRMS (ESI) calcd for $\mathrm{C}_{16} \mathrm{H}_{11} \mathrm{BrN}_{2} \mathrm{O}_{5}[\mathrm{M}+\mathrm{Na}]^{+} 412.9744$, found 412.9750 .

$(1 R, 2 R, 3 R)-2$-(4-Bromophenyl)-3-(2,4-dinitrophenyl)cyclopropane-1-carbaldehyde (4I). Yellow oil; The enantiomeric excess was determined by HPLC using a Chiralpak OD-H column (hexane $/ \mathrm{PrOH}=55: 45$, flow rate $=1.0 \mathrm{~mL} / \mathrm{min}, \lambda=210 \mathrm{~nm}$ ): $\mathrm{t}_{\mathrm{R}}=50.0 \mathrm{~min}, \mathrm{t}_{\mathrm{R}}=42.9$ $\min ;[\alpha]_{\mathrm{D}}{ }^{22}=+69.8^{\circ}\left(c 1.1, \mathrm{CHCl}_{3}\right)(\boldsymbol{R}$ catalyst $) ;{ }^{1} \mathrm{H} \mathrm{NMR}\left(400 \mathrm{MHz}, \mathrm{CDCl}_{3}\right) \delta=9.11(\mathrm{~d}, J=$ $5.0,1 \mathrm{H}), 8.83(\mathrm{~d}, J=2.3,1 \mathrm{H}), 8.43(\mathrm{dd}, J=8.6,2.3,1 \mathrm{H}), 7.54(\mathrm{~d}, J=8.6,1 \mathrm{H}), 7.49-7.46(\mathrm{~m}$, 2H), $7.26(\mathrm{~d}, J=8.3,2 \mathrm{H}), 3.94(\mathrm{dd}, J=7.1,5.5,1 \mathrm{H}), 3.13(\mathrm{dd}, J=9.3,7.3,1 \mathrm{H}), 2.62(\mathrm{ddd}, J$ = 9.6, 5.2, 4.4, 1H); ${ }^{13} \mathrm{C} \mathrm{NMR}\left(101 \mathrm{MHz}, \mathrm{CDCl}_{3}\right) \delta=196.6(\mathrm{CHO}), 150.1(\mathrm{Cq}), 146.9(\mathrm{Cq})$, $140.1(\mathrm{Cq}), 132.4(\mathrm{Cq}), 132.0(2 \mathrm{CH}), 130.6(2 \mathrm{CH}), 130.3(\mathrm{CH}), 127.5(\mathrm{CH}), 122.1(\mathrm{Cq}), 120.6$ $(\mathrm{CH}), 37.9(\mathrm{CH}), 34.5(\mathrm{CH}), 26.4(\mathrm{CH})$; HRMS (ESI) calcd for $\mathrm{C}_{16} \mathrm{H}_{11} \mathrm{BrN}_{2} \mathrm{O}_{5}[\mathrm{M}+\mathrm{Na}]^{+}$ 412.9744, found 412.9748 .

(1R,2S,3S)-2-(4-Bromophenyl)-3-(2,4-dinitrophenyl)cyclopropane-1-carbaldehyde (5l). Yellow oil; The enantiomeric excess was determined by HPLC using a Chiralpak OD-H column (hexane $/ i-\mathrm{PrOH}=55: 45$, flow rate $=1.0 \mathrm{~mL} / \mathrm{min}, \lambda=210 \mathrm{~nm}$ ): $\mathrm{t}_{\mathrm{R}}=30.6 \mathrm{~min}, \mathrm{t}_{\mathrm{R}}=34.0$ $\min ;[\alpha]_{\mathrm{D}}{ }^{22}=+46.8^{\circ}\left(c 0.2, \mathrm{CHCl}_{3}\right)(\boldsymbol{R}$ catalyst $) ;{ }^{1} \mathrm{H} \mathrm{NMR}\left(400 \mathrm{MHz}, \mathrm{CDCl}_{3}\right) \delta=9.71(\mathrm{~d}, J=$ $3.6,1 \mathrm{H}), 8.67(\mathrm{~d}, J=2.3,1 \mathrm{H}), 8.30(\mathrm{dd}, J=8.5,2.3,1 \mathrm{H}), 7.43(\mathrm{~d}, J=8.5,1 \mathrm{H}), 7.25-7.21(\mathrm{~m}$, 2H), $6.68(\mathrm{~d}, J=8.4,2 \mathrm{H}), 3.63(\mathrm{dd}, J=10.2,5.8,1 \mathrm{H}), 3.28(\mathrm{dd}, J=10.2,5.1,1 \mathrm{H}), 3.01-2.97$ $(\mathrm{m}, 1 \mathrm{H}) ;{ }^{13} \mathrm{C}$ NMR $\left(101 \mathrm{MHz}, \mathrm{CDCl}_{3}\right) \delta=197.3(\mathrm{CHO}), 150.4(\mathrm{Cq}), 147.0(\mathrm{Cq}), 137.0(\mathrm{Cq})$, $132.8(\mathrm{CH}), 131.9(\mathrm{Cq}), 131.8(2 \mathrm{CH}), 129.2(2 \mathrm{CH}), 126.9(\mathrm{CH}), 121.7(\mathrm{Cq}), 120.4(\mathrm{CH}), 35.2$ $(\mathrm{CH}), 32.6(\mathrm{CH}), 31.5(\mathrm{CH})$; HRMS (ESI) calcd for $\mathrm{C}_{16} \mathrm{H}_{11} \mathrm{BrN}_{2} \mathrm{O}_{5}[\mathrm{M}+\mathrm{Na}]^{+} 412.9744$, found 412.9732 .

2-(2,4-Dinitrophenyl)-3-(p-tolyl)cyclopropane-1-carbaldehyde (3m, $4 \mathrm{~m}$ and $5 \mathrm{~m})$ : The title compound was synthesized according to general procedure A. The product was purified by column chromatography (hexane/EtOAc $=5: 1$ ), affording the title compound as a yellow oil; Diastereomeric ratios of $\mathbf{3 m : 4 m : 5 m}=3: 2: 1$; Enantiomeric exceses of $\mathbf{3 m : 4 m : 5 m}=98: 85: 96$; Total yield of 3m:4m:5m = 80\% (120 mg). 
$(1 S, 2 R, 3 R)$-2-(2,4-Dinitrophenyl)-3-(p-tolyl)cyclopropane-1-carbaldehyde (3m). Yellow oil; The enantiomeric excess was determined by HPLC using a Chiralpak OD-H column (hexane $/ i-\mathrm{PrOH}=60: 40$, flow rate $=1.0 \mathrm{~mL} / \mathrm{min}, \lambda=230 \mathrm{~nm}$ ): $\mathrm{t}_{\mathrm{R}}=29.2 \mathrm{~min}, \mathrm{t}_{\mathrm{R}}=40.1 \mathrm{~min}$; $[\alpha]_{\mathrm{D}}{ }^{22}=+11.3^{\circ}\left(c 0.6, \mathrm{CHCl}_{3}\right)(\boldsymbol{S}$ catalyst $),[\alpha]_{\mathrm{D}}{ }^{22}=-11.8^{\circ}\left(c 1.1, \mathrm{CHCl}_{3}\right)(\boldsymbol{R}$ catalyst $) ;{ }^{1} \mathrm{H}$ $\operatorname{NMR}\left(400 \mathrm{MHz}, \mathrm{CDCl}_{3}\right) \delta=9.62(\mathrm{~d}, J=2.2,1 \mathrm{H}), 8.78(\mathrm{~d}, J=2.3,1 \mathrm{H}), 8.42(\mathrm{dd}, J=8.5,2.4$, $1 \mathrm{H}), 7.84(\mathrm{~d}, J=8.5,1 \mathrm{H}), 7.21-7.12(\mathrm{~m}, 4 \mathrm{H}), 3.42(\mathrm{dd}, J=8.2,7.2,1 \mathrm{H}), 3.28(\mathrm{dd}, J=7.5$, $5.1,1 \mathrm{H}), 2.97(\mathrm{ddd}, J=9.0,5.1,2.3,1 \mathrm{H}), 2.35(\mathrm{~s}, 3 \mathrm{H}) ;{ }^{13} \mathrm{C} \mathrm{NMR}\left(101 \mathrm{MHz}, \mathrm{CDCl}_{3}\right) \delta=197.2$ (CHO), $149.9(\mathrm{Cq}), 147.0(\mathrm{Cq}), 138.5(\mathrm{Cq}), 137.6(\mathrm{Cq}), 134.0(\mathrm{Cq}), 133.8(\mathrm{CH}), 129.7(2 \mathrm{CH})$, $127.1(\mathrm{CH}), 126.5(2 \mathrm{CH}), 120.1(\mathrm{CH}), 39.1(\mathrm{CH}), 34.1(\mathrm{CH}), 33.3(\mathrm{CH}), 21.1\left(\mathrm{CH}_{3}\right)$; IR $v_{\max }$ $\left(\mathrm{KBr}, \mathrm{cm}^{-1}\right.$ ): 3020, 2923, 2853, 1699, 1603, 1525 (aromatic $\mathrm{NO}_{2}$ ), 1434, 1397, 1342 (aroamatic $\mathrm{NO}_{2}$ ), 1215, 1150, 1127, 1065, 1038, 1010, 964, 909, 835, 802, 752, 738, 668; HRMS (ESI) calcd for $\mathrm{C}_{17} \mathrm{H}_{14} \mathrm{~N}_{2} \mathrm{O}_{5}[\mathrm{M}+\mathrm{Na}]^{+} 349.0795$, found 349.0797 .

$(1 R, 2 R, 3 R)$-2-(2,4-Dinitrophenyl)-3-(p-tolyl)cyclopropane-1-carbaldehyde $(4 \mathrm{~m})$. Yellow oil; The enantiomeric excess was determined by HPLC using a Chiralpak OD-H column (hexane $/ i-\mathrm{PrOH}=60: 40$, flow rate $=1.0 \mathrm{~mL} / \mathrm{min}, \lambda=230 \mathrm{~nm}$ ): $\mathrm{t}_{\mathrm{R}}=40.6 \mathrm{~min}, \mathrm{t}_{\mathrm{R}}=46.4 \mathrm{~min}$; $[\alpha]_{\mathrm{D}}{ }^{23}=+50.4^{\circ}\left(c 0.4, \mathrm{CHCl}_{3}\right)(\boldsymbol{R}$ catalyst $) ;{ }^{1} \mathrm{H} \mathrm{NMR}\left(400 \mathrm{MHz}, \mathrm{CDCl}_{3}\right) \delta=9.01(\mathrm{~d}, J=5.6$, $1 \mathrm{H}), 8.80(\mathrm{t}, J=2.1,1 \mathrm{H}), 8.41(\mathrm{dd}, J=8.6,2.4,1 \mathrm{H}), 7.54$ (d, $J=8.6,1 \mathrm{H}), 7.26(\mathrm{~d}, J=8.0$, 2H), $7.14(\mathrm{~d}, J=7.9,2 \mathrm{H}), 3.94(\mathrm{dd}, J=7.0,5.3,1 \mathrm{H}), 3.20(\mathrm{dd}, J=9.0,7.5,1 \mathrm{H}), 2.52$ (ddd, $J$ $=9.4,5.4,4.0,1 \mathrm{H}), 2.32(\mathrm{~s}, 3 \mathrm{H}) ;{ }^{13} \mathrm{C} \mathrm{NMR}\left(101 \mathrm{MHz}, \mathrm{CDCl}_{3}\right) \delta=197.5(\mathrm{CHO}), 150.1(\mathrm{Cq})$, $146.8(\mathrm{Cq}), 140.7(\mathrm{Cq}), 137.9(\mathrm{Cq}), 130.4(\mathrm{Cq}), 130.2(\mathrm{CH}), 129.6(2 \mathrm{CH}), 128.7(2 \mathrm{CH}), 127.5$ $(\mathrm{CH}), 120.5(\mathrm{CH}), 38.3(\mathrm{CH}), 34.7(\mathrm{CH}), 26.3(\mathrm{CH}), 21.1\left(\mathrm{CH}_{3}\right)$; IR $v_{\max }\left(\mathrm{KBr}, \mathrm{cm}^{-1}\right)$ : 3020, 2923, 2853, 1699, 1603, 1525 (aromatic $\mathrm{NO}_{2}$ ), 1434, 1397, 1342 (aroamtic $\mathrm{NO}_{2}$ ), 1215, 1150, 1127, 1065, 1038, 1010, 964, 909, 835, 802, 752, 738, 668; HRMS (ESI) calcd for $\mathrm{C}_{17} \mathrm{H}_{14} \mathrm{~N}_{2} \mathrm{O}_{5}$ $[\mathrm{M}+\mathrm{Na}]^{+}$349.0795, found 349.0803.

$(1 R, 2 S, 3 S)-2-(2,4-D i n i t r o p h e n y l)-3-(p$-tolyl)cyclopropane-1-carbaldehyde (5m). Yellow oil; The enantiomeric excess was determined by HPLC using a Chiralpak OD-H column (hexane $/ i-\mathrm{PrOH}=70: 30$, flow rate $=1.0 \mathrm{~mL} / \mathrm{min}, \lambda=230 \mathrm{~nm}$ ): $\mathrm{t}_{\mathrm{R}}=33.3 \mathrm{~min}, \mathrm{t}_{\mathrm{R}}=39.2 \mathrm{~min}$; $[\alpha]_{\mathrm{D}}{ }^{23}=-86.5^{\circ}\left(c 0.1, \mathrm{CHCl}_{3}\right)(S$ catalyst $),[\alpha]_{\mathrm{D}}{ }^{23}=+83.0^{\circ}\left(c 0.1, \mathrm{CHCl}_{3}\right)(\boldsymbol{R}$ catalyst $) ;{ }^{1} \mathrm{H}$ $\operatorname{NMR}\left(400 \mathrm{MHz}, \mathrm{CDCl}_{3}\right) \delta=9.66(\mathrm{~d}, J=3.9,1 \mathrm{H}), 8.62(\mathrm{~d}, J=2.4,1 \mathrm{H}), 8.25(\mathrm{dd}, J=8.5,2.4$, $1 \mathrm{H}), 7.43(\mathrm{~d}, J=8.5,1 \mathrm{H}), 6.90(\mathrm{t}, J=8.1,1 \mathrm{H}), 6.66(\mathrm{~d}, J=8.1,1 \mathrm{H}), 3.60(\mathrm{dd}, J=10.2,5.7$, $1 \mathrm{H}), 3.28(\mathrm{dd}, J=10.2,5.1,1 \mathrm{H}), 2.99-2.96(\mathrm{~m}, 1 \mathrm{H}), 2.20(\mathrm{~s}, 1 \mathrm{H}) ;{ }^{13} \mathrm{C}$ NMR $(101 \mathrm{MHz}$, $\left.\mathrm{CDCl}_{3}\right) \delta=197.9(\mathrm{CHO}), 150.4(\mathrm{Cq}), 146.8(\mathrm{Cq}), 137.7(\mathrm{Cq}), 137.4(\mathrm{Cq}), 133.0(\mathrm{CH}), 129.6$ $(\mathrm{Cq}), 129.3(2 \mathrm{CH}), 127.5(2 \mathrm{CH}), 126.7(\mathrm{CH}), 120.2(\mathrm{CH}), 35.5(\mathrm{CH}), 33.0(\mathrm{CH}), 31.3(\mathrm{CH})$, 
$21.0\left(\mathrm{CH}_{3}\right)$; IR $v_{\max }\left(\mathrm{KBr}, \mathrm{cm}^{-1}\right): 3020,2923,2853,1699,1603,1525$ (aromatic $\left.\mathrm{NO}_{2}\right), 1434$, 1397, 1342 (aromatic $\mathrm{NO}_{2}$ ), 1215, 1150, 1127, 1065, 1038, 1010, 964, 909, 835, 802, 752, 738, 668; HRMS (ESI) calcd for $\mathrm{C}_{17} \mathrm{H}_{14} \mathrm{~N}_{2} \mathrm{O}_{5}[\mathrm{M}+\mathrm{Na}]^{+} 349.0795$, found 349.0799.

\section{2-(2,4-Dinitrophenyl)-3-(4-methoxyphenyl)cyclopropane-1-carbaldehyde (3n, $4 \mathrm{n}$ and 5n):}

The title compound was synthesized according to general procedure A. The product was purified by column chromatography (hexane/EtOAc $=9: 1$ ), affording the title compound as a yellow oil; Diastereomeric ratios of $\mathbf{3 n}: \mathbf{4 n}: \mathbf{5 n}=1.5: 1: 1$; Enantiomeric exceses of $\mathbf{3 n}: \mathbf{4 n}: \mathbf{5 n}=$ 98:90:94; Total yield of 3n:4n:5n = 79\% (125 mg).

(1R,2S,3S)-2-(2,4-Dinitrophenyl)-3-(4-methoxyphenyl)cyclopropane-1-carbaldehyde

(3n). Yellow oil; The enantiomeric excess was determined by HPLC using a Chiralpak AD-H column (hexane $/ i-\mathrm{PrOH}=85: 15$, flow rate $=1.0 \mathrm{~mL} / \mathrm{min}, \lambda=245 \mathrm{~nm}$ ): $\mathrm{t}_{\mathrm{R}}=36.0 \mathrm{~min}, \mathrm{t}_{\mathrm{R}}=50.7$; $[\alpha]_{\mathrm{D}}{ }^{20}=+5.2^{\circ}\left(c 2.5, \mathrm{CHCl}_{3}\right)(\boldsymbol{S}$ catalyst $) ;{ }^{1} \mathrm{H}$ NMR $\left(500 \mathrm{MHz}, \mathrm{CDCl}_{3}\right) \delta=9.66(\mathrm{~d}, J=2.0$, $1 \mathrm{H}), 8.81(\mathrm{~d}, J=2.0,1 \mathrm{H}), 8.45(\mathrm{dd}, J=8.5,2.5,1 \mathrm{H}), 7.85(\mathrm{~d}, J=8.5,1 \mathrm{H}), 7.19$ (d, $J=8.5$, $2 \mathrm{H}), 6.91(\mathrm{~d}, J=9,2 \mathrm{H}), 3.82(\mathrm{~s}, 3 \mathrm{H}), 3.41(\mathrm{t}, J=8.5,1 \mathrm{H}), 3.29(\mathrm{dd}, J=7.5,5.0,1 \mathrm{H}), 2.96$ (ddd, $J=9.0,5.0,2.0,1 \mathrm{H}) ;{ }^{13} \mathrm{C} \mathrm{NMR}\left(125 \mathrm{MHz}, \mathrm{CDCl}_{3}\right) \delta=197.1,159.2,148.9,147.1,138.5$, 133.8, 128.9, 127.8 (2 C), 127.0, 120.1, 114.4 (2 C), 55.4, 39.1, 34.0, 33.0; IR $v_{\max }\left(\mathrm{KBr}, \mathrm{cm}^{-1}\right)$ : 3321, 2942, 2832, 1701, 1610, 1518 (aromatic $\mathrm{NO}_{2}$ ), 1450, 1348, 1249 (aromatic $\mathrm{NO}_{2}$ ), 1181, 1113, 1026, 909, 835, 737, 691, 672, 651, 635, 608; HRMS (ESI) calcd for $\mathrm{C}_{17} \mathrm{H}_{14} \mathrm{~N}_{2} \mathrm{O}_{6}$ $[\mathrm{M}+\mathrm{H}]^{+}$343.0925, found 343.0922.

(1S,2S,3S)-2-(2,4-Dinitrophenyl)-3-(4-methoxyphenyl)cyclopropane-1-carbaldehyde (4n). Yellow oil; The enantiomeric excess was determined by HPLC using a Chiralpak AD-H column (hexane $/ i-\mathrm{PrOH}=85: 15$, flow rate $=1.0 \mathrm{~mL} / \mathrm{min}, \lambda=245 \mathrm{~nm}$ ): $\mathrm{t}_{\mathrm{R}}=25.5 \mathrm{~min}, \mathrm{t}_{\mathrm{R}}=31.5$ $\min ;[\alpha]_{\mathrm{D}}{ }^{20}=-21^{\circ}\left(c 3.2, \mathrm{CHCl}_{3}\right)($ S catalyst $) ;{ }^{1} \mathrm{H} \mathrm{NMR}\left(500 \mathrm{MHz}, \mathrm{CDCl}_{3}\right) \delta=9.06(\mathrm{~d}, J=$ $5.5,1 \mathrm{H}), 8.84(\mathrm{~d}, J=2.5,1 \mathrm{H}), 8.44(\mathrm{dd}, J=8.5,2.0,1 \mathrm{H}), 7.54(\mathrm{~d}, J=8.5,1 \mathrm{H}), 7.32(\mathrm{~d}, J=$ $8.5,2 \mathrm{H}), 6.90(\mathrm{~d}, J=8.5,2 \mathrm{H}), 3.94(\mathrm{dd}, J=7.0,6.0,1 \mathrm{H}), 3.81(\mathrm{~s}, 3 \mathrm{H}), 3.19(\mathrm{dd}, J=9.0,5.0$, 1H), $2.54(\mathrm{~m}, 1 \mathrm{H}) ;{ }^{13} \mathrm{C} \mathrm{NMR}\left(125 \mathrm{MHz}, \mathrm{CDCl}_{3}\right) \delta=197.4,159.3,150.1,146.8,140.7,135.2$, 130 (2 C), 127.4, 125.3, 120.5, 114.3 (2 C), 55.3, 38.4, 34.5, 26.4; IR v $\max \left(\mathrm{KBr}, \mathrm{cm}^{-1}\right): 3321$, 2942, 2832, 1701, 1610, 1518 (aromatic $\mathrm{NO}_{2}$ ), 1450, 1348, 1249 (aromatic $\mathrm{NO}_{2}$ ), 1181, 1113, 1026, 909, 835, 737, 691, 672, 651, 635, 608; HRMS (ESI) calcd for $\mathrm{C}_{17} \mathrm{H}_{14} \mathrm{~N}_{2} \mathrm{O}_{6}[\mathrm{M}+\mathrm{H}]^{+}$ 343.0925, found 343.0927 .

(1S,2R,3R)-2-(2,4-Dinitrophenyl)-3-(4-methoxyphenyl)cyclopropane-1-carbaldehyde

(5n). Yellow oil; The enantiomeric excess was determined by HPLC using a Chiralpak AD-H 
column (hexane $/ i-\mathrm{PrOH}=85: 15$, flow rate $=1.0 \mathrm{~mL} / \mathrm{min}, \lambda=245 \mathrm{~nm}$ ): $\mathrm{t}_{\mathrm{R}}=45.2 \mathrm{~min}, \mathrm{t}_{\mathrm{R}}=55.0$ $\min ;[\alpha]_{\mathrm{D}}{ }^{20}=-20.7^{\circ}\left(c\right.$ 1.2, $\left.\mathrm{CHCl}_{3}\right)($ S catalyst $) ;{ }^{1} \mathrm{H} \mathrm{NMR}\left(500 \mathrm{MHz}, \mathrm{CDCl}_{3}\right) \delta=9.69(\mathrm{~d}, J=$ $4.0,1 \mathrm{H}), 8.66(\mathrm{~d}, J=2.0,1 \mathrm{H}), 8.28(\mathrm{dd}, J=8.5,2.0,1 \mathrm{H}), 7.42(\mathrm{~d}, J=8.5,1 \mathrm{H}), 6.73(\mathrm{~d}, J=$ $8.5,2 \mathrm{H}), 6.65(\mathrm{~d}, J=8.5,2 \mathrm{H}), 3.71(\mathrm{~s}, 3 \mathrm{H}), 3.61(\mathrm{dd}, J=10.0,5.5,1 \mathrm{H}), 3.30(\mathrm{dd}, J=10.3,5.0$, $1 \mathrm{H}), 2.96(\mathrm{dd}, J=9.5,5.0,1 \mathrm{H}) ;{ }^{13} \mathrm{C} \mathrm{NMR}\left(125 \mathrm{MHz} \mathrm{CDCl}_{3}\right) \delta=197.7,158.9,150.5,146.8$, 137.7, 135.2, 132.9, 128.8 (2 C), 126.7, 120.2, 114.1 (2 C), 55.2, 35.5, 32.7, 31.1; IR $v_{\max }(\mathrm{KBr}$, $\mathrm{cm}^{-1}$ ): 3321, 2942, 2832, 1701, 1610, 1518 (aromatic $\mathrm{NO}_{2}$ ), 1450, 1348, 1249 (aromatic $\mathrm{NO}_{2}$ ), 1181, 1113, 1026, 909, 835, 737, 691, 672, 651, 635, 608; HRMS (ESI) calcd for $\mathrm{C}_{17} \mathrm{H}_{14} \mathrm{~N}_{2} \mathrm{O}_{6}$ $[\mathrm{M}+\mathrm{H}]^{+}$343.0925, found 343.0929.

\section{2-(2-Nitro-4-(trifluoromethyl)phenyl)-3-phenylcyclopropane-1-carbaldehyde (3o, 40 and}

5o): The title compound was synthesized according to general procedure $B$. The product was purified by column chromatography (hexane/EtOAc $=5: 1$ ), affording the title compound as a yellow oil; Diastereomeric ratios of 3o:4o:5o $=1.5: 1: 1$; Enantiomeric exceses of 3o:4o:5o= 99:90:90; Total yield of 3o:4o:5o $=47 \%(65 \mathrm{mg})$.

$(1 R, 2 S, 3 S)$-2-(2-Nitro-4-(trifluoromethyl)phenyl)-3-phenylcyclopropane-1-carbaldehyde

(3o). Yellow oil; The enantiomeric excess was determined by HPLC using a Chiralpak OD-H column (hexane $/ i-\mathrm{PrOH}=80: 20$, flow rate $=1.0 \mathrm{~mL} / \mathrm{min}, \lambda=230 \mathrm{~nm}$ ): $\mathrm{t}_{\mathrm{R}}=18.1 \mathrm{~min}, \mathrm{t}_{\mathrm{R}}=13.5$ $\min ;[\alpha]_{\mathrm{D}}{ }^{26}=-37.3^{\circ}\left(c 0.5, \mathrm{CHCl}_{3}\right)($ S catalyst $) ;{ }^{1} \mathrm{H} \mathrm{NMR}\left(400 \mathrm{MHz}, \mathrm{CDCl}_{3}\right) \delta=9.01(\mathrm{~d}, J=$ $5.8,1 \mathrm{H}), 8.26(\mathrm{~d}, J=1.1,1 \mathrm{H}), 7.84(\mathrm{dd}, J=8.3,1.4,1 \mathrm{H}), 7.50(\mathrm{~d}, J=7.7,1 \mathrm{H}), 7.37(\mathrm{~m}, 6 \mathrm{H})$, $6.71(\mathrm{dd}, J=16.0,7.7,1 \mathrm{H}), 3.95(\mathrm{dd}, J=6.7,5.5,1 \mathrm{H}), 3.19$ (dd, $J=9.3,7.3,1 \mathrm{H}), 2.49$ (ddd, $J=9.2,5.5,3.7,1 \mathrm{H}) ;{ }^{13} \mathrm{C} \mathrm{NMR}\left(101 \mathrm{MHz}, \mathrm{CDCl}_{3}\right) \delta=197.7(\mathrm{CHO}), 137.6(\mathrm{Cq}), 134.0(\mathrm{Cq})$, $133.8(\mathrm{Cq}), 131.3(\mathrm{CH}), 130.8(\mathrm{Cq}), 129.9$ (q, J=3.4Hz, CH), $129.1(\mathrm{CH}), 128.9(\mathrm{CH}), 128.6$ $(\mathrm{CH}), 128.5(\mathrm{CH}), 127.9(\mathrm{CH}), 124.0(\mathrm{CH}), 122.4(\mathrm{q}, \mathrm{J}=3.7 \mathrm{~Hz}, \mathrm{CH}), 37.9(\mathrm{CH}), 34.4(\mathrm{CH})$, $26.3(\mathrm{CH}) ;{ }^{19} \mathrm{~F}$ NMR $\left(376 \mathrm{MHz}, \mathrm{CDCl}_{3}\right) \delta-62.9,-62.9,-63.0 \mathrm{ppm}$; IR $v_{\max }\left(\mathrm{KBr}, \mathrm{cm}^{-1}\right): 2923$, 2851, 2208, 2146, 2054, 2041, 2030, 2008, 1993, 1965, 1709, 1677, 1628, 1576, 1538, 1499, 1450, 1354, 1327 (aromatic $\mathrm{NO}_{2}$ ), 1134, 1091, 973, 908, 845, 813, 787, 751, 699, 644; HRMS (ESI) calcd for $\mathrm{C}_{17} \mathrm{H}_{12} \mathrm{~F}_{3} \mathrm{NO}_{3}[\mathrm{M}+\mathrm{H}]^{+} 336.0842$, found 336.0840 .

(1S,2S,3S)-2-(2-Nitro-4-(trifluoromethyl)phenyl)-3-phenylcyclopropane-1-carbaldehyde

(4o). Yellow oil; The enantiomeric excess was determined by HPLC using a Chiralpak OD-H column (hexane $/ i-\mathrm{PrOH}=70: 30$, flow rate $=1.0 \mathrm{~mL} / \mathrm{min}, \lambda=230 \mathrm{~nm}$ ): $\mathrm{t}_{\mathrm{R}}=13.5 \mathrm{~min}, \mathrm{t}_{\mathrm{R}}=11.6$ $\min ;[\alpha]_{\mathrm{D}}{ }^{26}=-52.9^{\circ}\left(c 0.4, \mathrm{CHCl}_{3}\right)(S$ catalyst $) ;{ }^{19} \mathrm{~F} \mathrm{NMR}\left(376 \mathrm{MHz}, \mathrm{CDCl}_{3}\right) \delta-63.0 \mathrm{ppm}$; ${ }^{1} \mathrm{H}$ NMR $\left(400 \mathrm{MHz}, \mathrm{CDCl}_{3}\right) \delta=9.01(\mathrm{~d}, J=5.8,1 \mathrm{H}), 8.26(\mathrm{~d}, J=1.1,1 \mathrm{H}), 7.84(\mathrm{dd}, J=8.1$, 
$1.4,1 \mathrm{H}), 7.50(\mathrm{~d}, J=8.2,1 \mathrm{H}), 7.37(\mathrm{ddd}, J=12.9,7.4,4.1,5 \mathrm{H}), 3.95(\mathrm{dd}, J=6.9,5.7,1 \mathrm{H})$, $3.19(\mathrm{dd}, J=9.2,7.3,1 \mathrm{H}), 2.49(\mathrm{dt}, J=9.4,5.5,1 \mathrm{H}) ;{ }^{13} \mathrm{C} \mathrm{NMR}\left(101 \mathrm{MHz}, \mathrm{CDCl}_{3}\right) \delta=197.7$ $(\mathrm{CHO}), 150.2(\mathrm{Cq}), 137.6(\mathrm{Cq}), 133.8(\mathrm{Cq}), 129.9$ ((q, J=3.4Hz, CH), $129.8(\mathrm{CH}), 129.5$ (q, $\mathrm{J}=33.9 \mathrm{~Hz}, \mathrm{Cq}), 129.2(\mathrm{Cq}), 128.9(\mathrm{CH}), 128.9(\mathrm{CH}), 127.9(\mathrm{CH}), 122.4$ (q, J=3.8Hz, CH), 37.9 (CH), $34.4(\mathrm{CH}), 26.3(\mathrm{CH})$; IR $v_{\max }\left(\mathrm{KBr}, \mathrm{cm}^{-1}\right)$ : 2923, 2851, 2208, 2146, 2054, 2041, 2030, 2008, 1993, 1965, 1709, 1677, 1628, 1576, 1538, 1499, 1450, 1354, 1327 (aromatic $\mathrm{NO}_{2}$ ), 1134, 1091, 973, 908, 845, 813, 787, 751, 699, 644; HRMS (ESI) calcd for $\mathrm{C}_{17} \mathrm{H}_{12} \mathrm{~F}_{3} \mathrm{NO}_{3}$ $[\mathrm{M}+\mathrm{H}]^{+}$336.0842, found: 336.0842 .

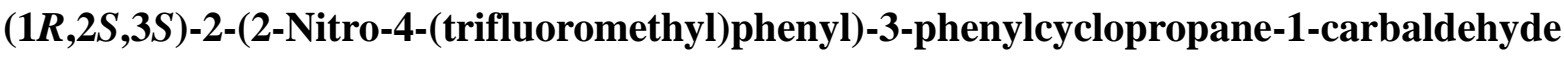

(5o). Yellow oil; The enantiomeric excess was determined by HPLC using a Chiralpak OD-H column (hexane $/ i-\mathrm{PrOH}=70: 30$, flow rate $=1.0 \mathrm{~mL} / \mathrm{min}, \lambda=230 \mathrm{~nm}$ ): $\mathrm{t}_{\mathrm{R}}=10.7 \mathrm{~min}, \mathrm{t}_{\mathrm{R}}=15.0$ $\min ;[\alpha]_{\mathrm{D}}{ }^{26}=+70.9^{\circ}\left(c 0.5, \mathrm{CHCl}_{3}\right)(\boldsymbol{R}$ catalyst $) ;{ }^{19} \mathrm{~F} \mathrm{NMR}\left(376 \mathrm{MHz}, \mathrm{CDCl}_{3}\right) \delta-63.0 \mathrm{ppm}$; ${ }^{1} \mathrm{H}$ NMR (400 MHz, $\left.\mathrm{CDCl}_{3}\right) \delta=9.67(\mathrm{~d}, J=3.9,1 \mathrm{H}), 8.05$ (s, 1H), $7.69(\mathrm{~d}, J=7.6,1 \mathrm{H}), 7.38$ (d, $J=8.1,1 \mathrm{H}), 7.12-7.05(\mathrm{~m}, 3 \mathrm{H}), 6.77$ (d, $J=7.8,2 \mathrm{H}), 3.62(\mathrm{dd}, J=10.1,5.7,1 \mathrm{H}), 3.28$ $(\mathrm{dd}, J=10.2,5.1,1 \mathrm{H}), 2.94(\mathrm{ddd}, J=9.5,5.3,3.9,1 \mathrm{H}) ;{ }^{13} \mathrm{C} \mathrm{NMR}\left(101 \mathrm{MHz}, \mathrm{CDCl}_{3}\right) \delta=198.0$ (CHO), $150.5(\mathrm{Cq}), 134.5(\mathrm{Cq}), 133.1(\mathrm{Cq}), 132.8(\mathrm{CH}), 130.9$ ((q, J=34.4Hz, Cq), 129.2 ((q, $\mathrm{J}=3.8 \mathrm{~Hz}, \mathrm{CH}), 128.5(\mathrm{CH}), 127.6(\mathrm{CH}), 127.4(\mathrm{CH}), 122.5$ (q, J=272 Hz, CF3), 122.0 ((q, $\mathrm{J}=3.8 \mathrm{~Hz}, \mathrm{CH}), 35.7(\mathrm{CH}), 32.8(\mathrm{CH}), 31.5(\mathrm{CH})$; IR $v_{\max }\left(\mathrm{KBr}, \mathrm{cm}^{-1}\right)$ : 2923, 2851, 2208, 2146, 2054, 2041, 2030, 2008, 1993, 1965, 1709, 1677, 1628, 1576, 1538, 1499, 1450, 1354, 1327 (aromatic $\mathrm{NO}_{2}$ ), 1134, 1091, 973, 908, 845, 813, 787, 751, 699, 644; HRMS (ESI) calcd for $\mathrm{C}_{17} \mathrm{H}_{12} \mathrm{~F}_{3} \mathrm{NO}_{3}[\mathrm{M}+\mathrm{Na}]^{+} 358.0661$, found 358.0668.

\section{2-(2-Nitro-4-(trifluoromethyl)phenyl)-3-(4-nitrophenyl)cyclopropane-1-carbaldehyde}

(3p, $4 p$ and 5p): The title compound was synthesized according to general procedure $B$. The product was purified by column chromatography (hexane/EtOAc $=7: 1$ ), affording the title

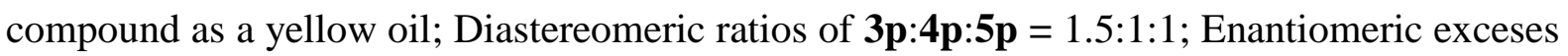
of 3p:4p:5p = 85:n.d.:79; Total yield of 3p:4p:5p = 52\% $(82 \mathrm{mg})$.

(1S,2R,3R)-2-(2-nitro-4-(trifluoromethyl)phenyl)-3-(4-nitrophenyl)cyclopropane-1-

carbaldehyde (3p). Yellow oil; The enantiomeric excess was determined by HPLC using a Chiralpak OD-H column (hexane $/ i-\mathrm{PrOH}=75: 25$, flow rate $=1.0 \mathrm{~mL} / \mathrm{min}, \lambda=230 \mathrm{~nm}$ ): $\mathrm{t}_{\mathrm{R}}=$ $20.4 \mathrm{~min}, \mathrm{t}_{\mathrm{R}}=27.7 \mathrm{~min} ;[\alpha]_{\mathrm{D}}{ }^{23}=-11.0^{\circ}\left(c 0.5, \mathrm{CHCl}_{3}\right)(\boldsymbol{R}$ catalyst $) ;{ }^{1} \mathrm{H} \mathrm{NMR}\left(400 \mathrm{MHz}, \mathrm{CDCl}_{3}\right)$ $\delta=9.61(\mathrm{~d}, J=2.2,1 \mathrm{H}), 8.27-8.21(\mathrm{~m}, 3 \mathrm{H}), 7.87(\mathrm{~d}, J=7.1,1 \mathrm{H}), 7.72(\mathrm{~d}, J=8.1,1 \mathrm{H}), 7.41$ $(\mathrm{d}, J=8.7,2 \mathrm{H}), 3.49(\mathrm{t}, J=8.3,1 \mathrm{H}), 3.38(\mathrm{dd}, J=7.2,5.3,1 \mathrm{H}), 3.06(\mathrm{ddd}, J=9.2,5.1,2.3$, 
1H); ${ }^{13} \mathrm{C}$ NMR $\left(101 \mathrm{MHz}, \mathrm{CDCl}_{3}\right) \delta=196.0(\mathrm{CHO}), 149.9(\mathrm{Cq}), 147.3(\mathrm{Cq}), 145.0(\mathrm{Cq}), 134.1$ $(\mathrm{Cq}), 133.2(\mathrm{CH}), 131.5$ (q, J=34.4Hz, Cq), 129.7 (q J=3.4hz, CH) q, $127.4(\mathrm{CH}), 124.2(\mathrm{CH})$, 122.2 (q J=3.8Hz, CH), $121.3(\mathrm{Cq}), 39.0(\mathrm{CH}), 34.6(\mathrm{CH}), 31.8(\mathrm{CH}) ;{ }^{19} \mathrm{~F}$ NMR $(376 \mathrm{MHz}$, $\left.\mathrm{CDCl}_{3}\right) \delta-63.0$ ppm; IR $v_{\max }\left(\mathrm{KBr}, \mathrm{cm}^{-1}\right): 2922,2851,1706,1629,1601,1537$ (aromatic $\mathrm{NO}_{2}$ ), 1519, 1346, 1325 (aromatic $\mathrm{NO}_{2}$ ), 1214, 1180, 1136, 1094, 1013, 960, 853, 746, 668; HRMS (ESI) calcd for $\mathrm{C}_{17} \mathrm{H}_{11} \mathrm{~F}_{3} \mathrm{~N}_{2} \mathrm{O}_{5}[\mathrm{M}+\mathrm{Na}]^{+}$403.0512, found 403.0507.

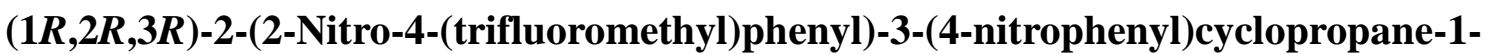

carbaldehyde (4p). Yellow oil; $[\alpha]_{\mathrm{D}}{ }^{23}=66.3^{\circ}\left(c 0.2, \mathrm{CHCl}_{3}\right)(\boldsymbol{R}$ catalyst $) ;{ }^{19} \mathrm{~F} \mathrm{NMR}(376 \mathrm{MHz}$, $\left.\mathrm{CDCl}_{3}\right) \delta-63.0 \mathrm{ppm} ;{ }^{1} \mathrm{H} \mathrm{NMR}\left(400 \mathrm{MHz}, \mathrm{CDCl}_{3}\right) \delta=9.27(\mathrm{~d}, J=4.2,1 \mathrm{H}), 8.29(\mathrm{~d}, J=1.2$, $1 \mathrm{H}), 8.21(\mathrm{~d}, J=8.8,2 \mathrm{H}), 7.87(\mathrm{dd}, J=8.2,1.7,1 \mathrm{H}), 7.57(\mathrm{~d}, J=8.4,2 \mathrm{H}), 7.53(\mathrm{~d}, J=8.1$, $1 \mathrm{H}), 4.05-3.99(\mathrm{~m}, 1 \mathrm{H}), 3.16(\mathrm{dd}, J=9.4,7.5,1 \mathrm{H}), 2.75(\mathrm{ddd}, J=9.6,5.5,4.3,1 \mathrm{H}) ;{ }^{13} \mathrm{C} \mathrm{NMR}$ $\left(101 \mathrm{MHz}, \mathrm{CDCl}_{3}\right) \delta=196.0(\mathrm{CHO}), 150.1(\mathrm{Cq}), 147.4(\mathrm{Cq}), 141.2(\mathrm{Cq}), 136.6(\mathrm{Cq}), 131.5(\mathrm{q}$, $\mathrm{J}=34.6 \mathrm{~Hz}, \mathrm{Cq}), 130.3(\mathrm{CH}), 130.1$ (q, J=3.5Hz, CH), $130.0(\mathrm{CH}), 129.9(\mathrm{CH}), 123.9(\mathrm{CH})$, 122.6 (q, J=3.8Hz, CH), $121.2(\mathrm{Cq}), 37.5(\mathrm{CH}), 34.6(\mathrm{CH}), 27.1(\mathrm{CH}) ; \mathrm{IR} v_{\max }\left(\mathrm{KBr}, \mathrm{cm}^{-1}\right)$ : 2922, 2851, 1706, 1629, 1601, 1537 (aromatic $\mathrm{NO}_{2}$ ), 1519, 1346, 1325 (aromatic $\mathrm{NO}_{2}$ ), 1214, 1180, 1136, 1094, 1013, 960, 853, 746, 668; HRMS (ESI) calcd for $\mathrm{C}_{17} \mathrm{H}_{11} \mathrm{~F}_{3} \mathrm{~N}_{2} \mathrm{O}_{5}[\mathrm{M}+\mathrm{Na}]^{+}$ 403.0512, found 403.0503.

$(1 R, 2 S, 3 S)$-2-(2-Nitro-4-(trifluoromethyl)phenyl)-3-(4-nitrophenyl)cyclopropane-1-

carbaldehyde (5p). Yellow oil; The enantiomeric excess was determined by HPLC using a Chiralpak OD-H column (hexane $/ i-\mathrm{PrOH}=70: 30$, flow rate $=1.0 \mathrm{~mL} / \mathrm{min}, \lambda=210 \mathrm{~nm}$ ): $\mathrm{t}_{\mathrm{R}}=$ $24.7 \mathrm{~min}, \mathrm{t}_{\mathrm{R}}=28.5 \mathrm{~min} ;[\alpha]_{\mathrm{D}}{ }^{23}=-18.2^{\circ}\left(c 0.5, \mathrm{CHCl}_{3}\right)(\boldsymbol{R}$ catalyst $) ;{ }^{1} \mathrm{H} \mathrm{NMR}(400 \mathrm{MHz}$, $\left.\mathrm{CDCl}_{3}\right) \delta=9.75(\mathrm{~d}, J=3.5,1 \mathrm{H}), 8.08(\mathrm{~d}, J=1.2,1 \mathrm{H}), 7.96(\mathrm{~d}, J=8.8,2 \mathrm{H}), 7.76(\mathrm{dd}, J=8.1$, $1.5,1 \mathrm{H}), 7.48(\mathrm{~d}, J=8.1,1 \mathrm{H}), 6.96(\mathrm{~d}, J=8.7,2 \mathrm{H}), 3.70(\mathrm{dd}, J=10.2,5.9,1 \mathrm{H}), 3.36(\mathrm{dd}, J=$ $10.2,5.2,1 \mathrm{H}), 3.07(\mathrm{td}, J=5.5,3.5,1 \mathrm{H}) ;{ }^{13} \mathrm{C} \mathrm{NMR}\left(101 \mathrm{MHz}, \mathrm{CDCl}_{3}\right) \delta=197.0(\mathrm{CHO}), 150.2$ $(\mathrm{Cq}), 147.1(\mathrm{Cq}), 141.2(\mathrm{Cq}), 133.3(\mathrm{Cq}), 132.7(\mathrm{CH}), 131.5$ (q, J=35Hz, Cq), 129. (q, J=3.4Hz, $\mathrm{CH}), 128.4(\mathrm{CH}), 123.7(\mathrm{CH}), 122.4(\mathrm{q}, \mathrm{J}=3.7 \mathrm{~Hz}, \mathrm{CH}), 121.1(\mathrm{Cq}), 35.7(\mathrm{CH}), 32.4(\mathrm{CH}), 32.4$ $(\mathrm{CH}) ;{ }^{19} \mathrm{~F}$ NMR $\left(376 \mathrm{MHz}, \mathrm{CDCl}_{3}\right) \delta-63.1 \mathrm{ppm}$; IR $v_{\max }\left(\mathrm{KBr}, \mathrm{cm}^{-1}\right): 2922,2851,1706,1629$, 1601, 1537 (aromatic $\mathrm{NO}_{2}$ ), 1519, 1346, 1325 (aromatic $\mathrm{NO}_{2}$ ), 1214, 1180, 1136, 1094, 1013, 960, 853, 746, 668; HRMS (ESI) calcd for $\mathrm{C}_{17} \mathrm{H}_{11} \mathrm{~F}_{3} \mathrm{~N}_{2} \mathrm{O}_{5}[\mathrm{M}+\mathrm{Na}]^{+} 403.0512$, found 403.0515 .

General procedure for ring-opening of cyclopropanation product $(3 \mathrm{~g})$ :

To a suspension of thiazolium precatalyst 9 (7.9 $\mathrm{mg}, 0.033 \mathrm{mmol}, 20 \mathrm{~mol} \%)$ in $\mathrm{CH}_{2} \mathrm{Cl}_{2}(0.5$ 
$\mathrm{mL}$ ) was added the cyclopropanation product $(\mathbf{3 g} ; 52 \mathrm{mg}, 0.166 \mathrm{mmol}), \mathrm{MeOH}(20 \mu \mathrm{L}, 0.5$ $\mathrm{mmol}$ ), and $N, N$-diisopropylethylamine (DIPEA; $11 \mu \mathrm{L}, 0.06 \mathrm{mmol}$ ) at room temperature. The resulting solution was stirred for 6 hours. The crude product was purified by flash column chromatography on silica gel (hexane/EtOAc $=6: 1)$ to afford the final ester $\mathbf{1 0}(48.3 \mathrm{mg}, 84 \%)$.

Methyl (S)-4-(2,4-dinitrophenyl)-3-phenylbutanoate (10). The title compound was synthesized according to the above-mentioned procedure. Yellow oil; The enantiomeric excess was determined by HPLC using a Chiralpak OD-H column (hexane $/ i$-PrOH $=90: 10$, flow rate $=1.0 \mathrm{~mL} / \mathrm{min}, \lambda=245 \mathrm{~nm}): \mathrm{t}_{\mathrm{R}}=26.6 \mathrm{~min}, \mathrm{t}_{\mathrm{R}}=37.7 \mathrm{~min} ;[\alpha]_{\mathrm{D}}{ }^{23}=-32^{\circ}\left(c 0.59, \mathrm{CHCl}_{3}\right) ;{ }^{1} \mathrm{H}$ NMR $\left(500 \mathrm{MHz}, \mathrm{CDCl}_{3}\right) \delta=8.68(\mathrm{~d}, J=2.5,1 \mathrm{H}), 8.19-8.16(\mathrm{dd}, J=8.5,2.0,1 \mathrm{H}), 7.27-7.19$ (m, 4H), 7.05-7.04 (m, 2H), $3.61(\mathrm{~s}, 1 \mathrm{H}), 3.53-3.46(\mathrm{~m}, 2 \mathrm{H}), 3.28-3.23(\mathrm{~m}, 1 \mathrm{H}), 2.80-2.78(\mathrm{~d}$, $J=7,2 \mathrm{H}) ;{ }^{13} \mathrm{C}$ NMR $\left(125 \mathrm{MHz}, \mathrm{CDCl}_{3}\right) \delta=171.9,149.6,146.5,141.7,141.3,134.1,128.9$ (2 C), 127.5, 127.5 (2 C), 126.4, 120.3, 51.9, 43.3, 40.7, 39.5; IR $v_{\max }\left(\mathrm{KBr}, \mathrm{cm}^{-1}\right): 3708,3322$, 2977. 2946, 2884, 2851, 2363, 2339, 2323, 2059, 1737, 1605, 1539, 1350, 1160, 658; HRMS (ESI) calcd for $\mathrm{C}_{17} \mathrm{H}_{16} \mathrm{~N}_{2} \mathrm{O}_{6}[\mathrm{M}+\mathrm{H}]^{+} 345.1081$, found 345.1070.

\section{- ASSOCIATED CONTENT}

\section{Supporting Information}

The Supporting Information is available free of charge on the ACS Publications website at DOI:

Copies of ${ }^{1} \mathrm{H}$ NMR and ${ }^{13} \mathrm{C}$ NMR spectra, HRMS, and HPLC chromatograms of all compounds $\mathrm{X}$-ray crystallographic file for ent-3i

X-ray crystallographic file for ent-31.

Determination of absolute and relative configuration of products $[(\mathbf{3 d}, \mathbf{4 d}, \mathbf{5 d})$ and $(\mathbf{3 k}, \mathbf{4 k}$, $5 \mathbf{k})]$.

\section{- AUTHOR INFORMATION}

\section{Corresponding Authors}

*E-mail: R.Rios-Torres@ southampton.ac.uk (R. Rios)

*E-mail: jwyang@skku.edu (J. W. Yang)

\section{Notes}


The authors declare no competing financial interest.

\section{- ACKNOWLEDGMENTS}

M.A., M.M. and R.R acknowledge the European Regional Development Fund (ERDF) for cofinancing the AI-CHEM-Chem project (No. 4061) through the INTERREG IV A France (Channel) - England cross-border cooperation Programme. M.A., M.M. and R.R. are grateful for the EPSRC Core Capability Funding (EP/ K039466/1). J.W.Y. thanks the NRF Nano·Material Technology Development Program (2012M3A7B4049652) for financial support.

\section{- REFERENCES}

(1) (a) Simmons, H. E.; Smith, R. D. J. Am. Chem. Soc. 1958, 80, 5323. (b) Simmons, H. E.; Smith, R. D. J. Am. Chem. Soc. 1959, 81, 4256.

(2) For an excellent review on asymmetric cyclopropanation, see: Pellissier, H. Tetrahedron 2008, 64, 7041.

(3) (a) List, B.; Lerner, R. A.; Barbas III, C. F. J. Am. Chem. Soc. 2000, 122, 2395. (b) Ahrendt, K. A.; Borths, C. J.; MacMillan, D. W. C. J. Am. Chem. Soc. 2000, 122, 4243.

(4) Papageorgiou, C. D.; Cubillo de Dios, M. A.; Ley, S. V.; Gaunt, M. J. Angew. Chem. Int. Ed. 2004, 43, 4641.

(5) McCooey, S. H.; McCabe, T.; Connon, S. J. J. Org. Chem. 2006, 71, 7494.

(6) Kunz, R. K.; MacMillan, D. W. C. J. Am. Chem. Soc. 2005, 127, 3240.

(7) (a) Rios, R.; Sundén, H.; Vesely, J.; Zhao, G.-L.; Dziedzic, P.; Córdova, A. Adv. Synth. Catal. 2007, 349, 1028. (b) Ibrahem, I.; Zhao, G.-L.; Rios, R.; Vesely, J.; Sundén, H.; Dziedzic, P.; Córdova, A. Chem. Eur. J. 2008, 14, 7867.

(8) Xie, H.; Zu, L.; Li, H.; Wang, J.; Wang, W. J. Am. Chem. Soc. 2007, 129, 10886.

(9) Russo, A.; Meninno, S.; Tedesco, C.; Lattanzi, A. Eur. J. Org. Chem. 2011, 5096.

(10) Li, T.; Zhu, J.; Wu, D.; Li, X.; Wang, S.; Li, H.; Li, J.; Wang, W. Chem. Eur. J. 2013, $19,9147$.

(11) Dell'Amico, L.; Companyó, X.; Naicker, T.; Bräuer, T. M.; Jørgensen, K. A. Eur. J. Org. Chem. 2013, 5262.

(12) Raja, A.; Hong, B.-C.; Lee, G.-H. Org. Lett. 2014, 16, 5756. 
(13) Meazza, M.; Ceban, V.; Pitak, M. B.; Coles, S. J.; Rios, R. Chem. Eur. J. 2014, 20, 16853.

(14) Arceo, E.; Jurberg, I. D.; Álvarez-Fernández, A.; Melchiorre, P. Nat. Chem. 2013, 5, 750.

(15) For examples of cyclopropanations developed by our group, see: (a) Meazza, M.; Light, M. E.; Mazzanti, A.; Rios, R. Chem. Sci. 2015, DOI: 10.1039/C5SC03597J. (b) Companyó, X.; Alba, A.-N.; Cárdenas, F.; Moyano, A.; Rios, R. Eur. J. Org. Chem. 2009, 3075.

(16) For reviews on organocascade reactions, see: (a) Moyano, A.; Rios, R. Chem. Rev. 2011, 111, 4703. (b) Alba, A.-N.; Companyó, X.; Viciano, M.; Rios, R. Curr. Org. Chem. 2009, 13, 1432. (c) Enders, D.; Grondal, C.; Hüttl, M. R. M. Angew. Chem. Int. Ed. 2007, 46, 1570.

(17) Sohn, S. S.; Bode, J. W. Angew. Chem. Int. Ed. 2006, 45, 6021. 